\title{
Thionated Naphthalene Diimides: Tuneable Chromophores for Applications in Photoactive Dyads
}

\author{
Nicholas Pearce, E. Stephen Davies, Raphael Horvath, Constance R. Pfeiffer, Xue-Zhong Sun, \\ William Lewis, Jonathon McMaster, Michael W. George and Neil R. Champness* \\ School of Chemistry, University of Nottingham, University Park, Nottingham NG7 2RD, UK. \\ E-mail: Neil.Champness@nottingham.ac.uk.
}

\begin{abstract}
Varying the degree of thionation of a series of naphthalene diimide (NDI) and naphthalic imides (NI) phenothiazine dyad systems afford a systematic approach for tuning of the system's donor-acceptor energy gap. Each dyad was compared to model NDI/NI systems and fully characterised through single crystal X-ray diffraction, NMR, cyclic voltammetry, electron paramagnetic resonance (EPR), transient absorption spectroscopy (TA), time-resolved infrared spectroscopy (TRIR) and DFT. The measurements reveal that thionation increases both electron affinity of the NDI/NI acceptor dyad component and accessibility of the singly or doubly reduced states. Furthermore, FTIR and TA measurements show that excited state behaviour is greatly affected by thionation of the NDI and induces a decrease in the lifetime of the excited states formed upon the creation of charge-separated states.
\end{abstract}

\section{Introduction}

Molecular dyads, molecules composed of both donor and acceptor groups, have been widely studied due to their relationship to photosynthetic organisms and their potential for photochemical energy conversion. ${ }^{1}$ For such dyad systems, focus has been on the modification of the molecular architecture with the aim to manipulate the energy differences between the HOMO and LUMO of the donor and acceptor moieties and to control the lifetimes of the excited charge-separated states. ${ }^{1-4}$ 
This study focusses on the use of naphthalene diimides (NDIs), and their thionated analogues, as electron acceptors in photochemical dyads. Reliable redox chemistry, strong fluorescence, and chemical adaptability of NDIs have led to their implementation in a variety of applications $^{5}$ such as organic electronics, ${ }^{6-8}$ photovoltaic devices, ${ }^{9-15}$ anion recognition, ${ }^{16-18}$ and synthesis of metal-organic supramolecular structures ${ }^{19}$. Unlike other acceptor molecules used in photovoltaic devices, for example fullerenes and perylene diimides, NDIs have a larger HOMO-LUMO energy gap that results in weak absorbance in the visible spectrum. ${ }^{20-22}$ In order to make NDIs more successful electron acceptors in photovoltaic devices, it is desirable to tune the electrochemical properties of NDIs and the related NIs. However, manipulation of the redox properties of NDIs/NIs is limited by the synthetic demands of chemical modification of the aromatic core. It is relatively straightforward to functionalize the imide nitrogen; however, this typically has little influence on the properties of the NDI/NI and more intensive chemical modification of the naphthalene core is often mandatory. ${ }^{23,24}$ Carbonyl groups of NDIs/NIs play a significant role in influencing the nature of the frontier orbitals of these species, therefore substitution with sulfur is likely to strongly affect the properties of these species. ${ }^{25-30}$ Studies by Seferos et al., amongst others, report that the reaction of NDIs ${ }^{25-27}$, and their analogues PDIs ${ }^{25,28-30}$, with Lawesson's reagent resulted in the substitution of the carbonyl oxygen atoms with sulfur atoms to give thionated dyes. Thionation was observed to have a clear effect on the redox properties of the NDIs, primarily increasing electron mobility. ${ }^{26,29}$

Due to the successful approach of using thionation to affect NDI redox properties, NDIs/NIs are excellent candidates for electron acceptors in donor-acceptor dyad systems. Thus, we have pursued the development of such systems in order to demonstrate the ability to tune the energy gap in molecular dyads. Phenothiazine (PTZ) was selected as the electron donor, inspired by promising results obtained by Wasielewski and co-workers for a related phenothiazine-perylene diimide system. ${ }^{31}$ A series of NDI/NI-phenothiazine donor-acceptor dyads with varying degrees of thionation was synthesised. Additionally, a series of NDI/NI compounds with variable degrees of thionation were prepared for comparison to the dyads. The dyads and model compounds were fully characterized by single crystal X-ray diffraction and NMR, cyclic voltammetry, spectroelectrochemistry, transient absorption (TA) and time resolved infra-red (TRIR) spectroscopy, electron paramagnetic resonance (EPR), and DFT calculations were used to study the electrochemical properties of each dyad and analogous 
$\mathrm{NDI} / \mathrm{NI}$ species and the effect of addition of sulfur atoms. It is demonstrated that the HOMOLUMO energy gap can be decreased through successive degrees of thionation, which ultimately culminates in a phenothiazine-NDI dyad with an unprecedentedly low optical gap for this class of compound.

\section{Results and Discussion}

\section{Synthesis and Structural Characterisation}

Thionation of NDIs was readily achieved using Lawesson's reagent leading to a number of products with different degrees of substitution (see SI for full experimental). In order to prepare the target dyads it was necessary to couple the phenothiazine to the selected acceptors, requiring furnishing of the phenothiazine with an amine moiety capable of reaction with the anhydride functionalities of the NI and NDI precursors. A phenylene spacer was used to separate the phenothiazine and amine over an aliphatic alternative, as this would allow for a more conformationally-defined architecture and decoupling of the dyad components. Thus, PTZ- $\mathrm{NH}_{2}$ was synthesised ${ }^{21}$ and coupled to either a $\mathrm{N}$-alkylated naphthalene monoimide

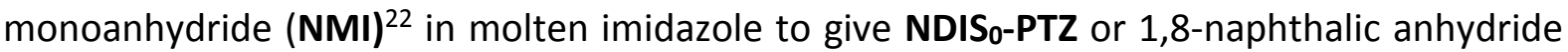
to prepare a naphthalic monoimide, NISo-PTZ. Although both nonthionated dyads form colourless dichloromethane solutions, NIS0-PTZ was isolated as a bright yellow solid and NDIS $_{0}-\mathbf{P T Z}$ as a blue solid. The NDI or NI fragment of NDIS $\mathbf{S}_{0}-\mathbf{P T Z}$ or $\mathbf{N I S}_{0}-\mathbf{P T Z}$ was thionated (Scheme 1) by subsequent reaction with Lawesson's reagent, resulting in a mixture of thionated species. Thionation of NDIS $\mathbf{S}_{0}$-PTZ resulted in a mixture of di- and tri-thionated products. Column chromatography of the crude mixture allowed for isolation of NDIS - PTZ and cis-NDIS $\mathbf{2}$-PTZ. Small quantities of other thionated species, including trans-NDIS $\mathbf{2}-\mathbf{P T Z}$ and gem-NDIS 2 -PTZ were separated from the reaction mixture, but were found to be an inseparable mixture of thionated NDI isomers. Full thionation of NIS $\mathbf{S}_{0}-\mathbf{P T Z}$ resulted in NIS $\mathbf{S}_{2}-\mathbf{P T Z}$ with an overall yield of $48 \%$.
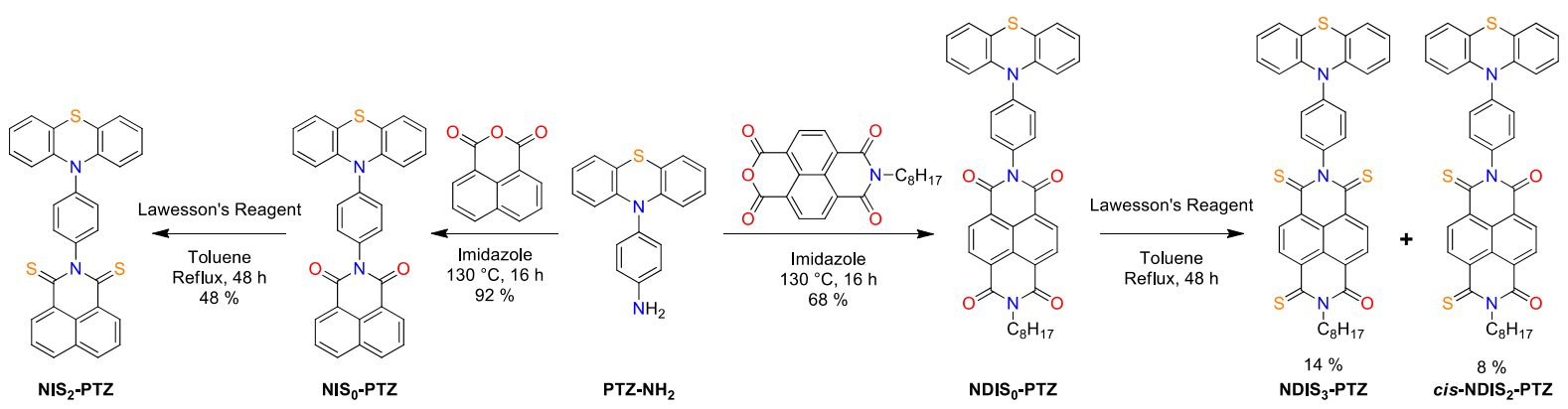

Scheme 1. Synthesis of thionated phenothiazine donor-acceptor molecules. 
A series of model compounds were prepared for direct comparison to the dyad molecules. Ethylpropyl-disubstituted NDI, NDIS , was used due to its enhanced solubility over related $n$-alkyl substituted NDIs. The reaction of NDIS $\mathbf{S}_{\mathbf{0}}$ with Lawesson's reagent in anhydrous toluene results in a significant change in colour of the reaction solution and subsequent workup and purification of the reaction solution enabled isolation of four separate thionated NDIs, with one (NDIS $)_{1}$, two (cis-NDIS 2 and trans-NDIS $\mathbf{S}_{\mathbf{2}}$ ) and three $\left(\mathbf{N D I S}_{\mathbf{3}}\right)$ of the imide carbonyls replaced with thiocarbonyls (Scheme 2). No evidence was found for any tetrathionated NDI, despite exploring a variety of reaction conditions including stoichiometry of Lawesson's reagent, reaction time, and solvent, which is consistent with previous observations. $^{25-27}$ Standard spectroscopic techniques allowed for identification of the thionated NDI products.
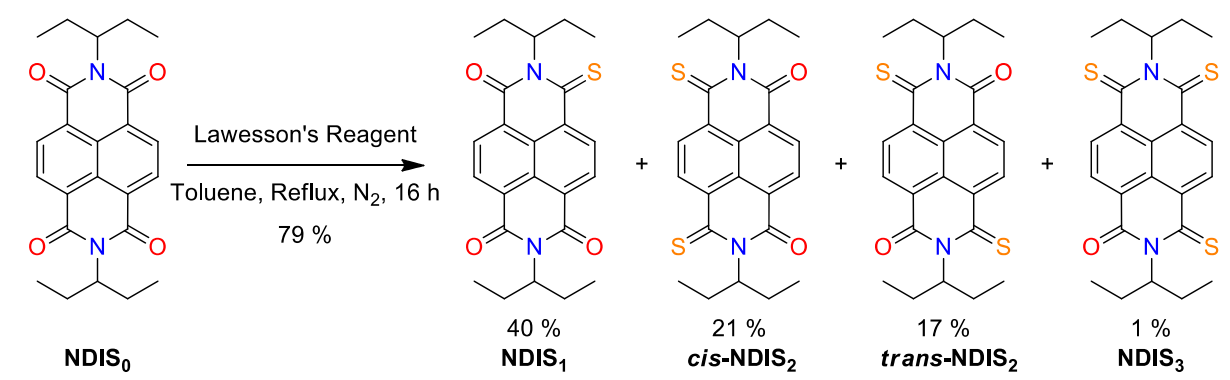

Scheme 2. Synthesis of a series of thionated NDIs using Lawesson's reagent.

The two isomeric dithionated isomers, cis-NDIS $\mathbf{2}$ and trans-NDIS $\mathbf{S}_{\mathbf{2}}$, were readily distinguishable by ${ }^{1} \mathrm{H}$ NMR spectroscopy. Although a further dithionated isomer containing a dithioimide functionality (i.e. the two sulfurs are part of the same imide moiety) is possible, this product was not observed, and this accounts for the low isolated yield of NDIS $\mathbf{3}(<1 \%)$. Moreover, it is worth noting that the mono- and dithionated compounds are stable to air and light unlike NDIS $_{3}$, which degraded over a period of hours and thus, had to be used immediately following purification. Model $\mathrm{NI}$ systems were also prepared and studied and in this case only the non-thionated species $\mathbf{N I S}_{\mathbf{0}}$ or the mono-thionated species $\mathbf{N I S}_{\mathbf{1}}$ were isolated. Again, the formation of a dithioimide moiety required for the production of $\mathbf{N I S}_{\mathbf{2}}$ was not observed. This is in direct contrast with the synthesis of NIS-PTZ, which was formed in good yield and with no monothionation product isolated, and NDIS - PTZ in which the phenyl- 
substituted imide is dithionated. Our results indicate that thionation of phenyl-substituted imides progresses more readily to dithionation than alkyl-substituted imide analogues.

Single crystal X-ray diffraction data was obtained for dyads NIS0-PTZ, NIS - -PTZ, NDIS0PTZ, and NDIS $\mathbf{3}-\mathbf{P T Z}$ and standards NDIS, $\mathbf{N D I S}_{\mathbf{1}}$, and trans-NDIS $\mathbf{2}$ to confirm molecular structure and the arrangement of sulfur substituents (Figure 1). As expected, carbonyl bonds in both NDIs and NIs are shorter than corresponding thiocarbonyl bonds (Table 1). Furthermore, in all dyad structures, conjugation between donor and acceptor is inhibited due to the phenylene spacer separating the phenothiazine and naphthalic imide group lying perpendicular to the NDI/NI plane. All three model compounds and both NI dyads pack in an analogous herringbone manner, regardless of the degree of thionation. For the NDI dyads systems the molecules are stacked, in a staggered fashion for NDIS $\mathbf{0}_{\mathbf{0}}$-PTZ, with phenothiazine moieties positioned adjacent to NDIs groups, in contrast to NDIS $\mathbf{S}_{3}-\mathrm{PTZ}$ for which $\mathrm{NDIS}_{3}-\mathrm{NDIS}_{3}$ stacking is observed (Figure 2).

Table 1. Selected bond lengths for dyads and related NDI species

\begin{tabular}{|c|c|c|c|c|}
\hline Bond Lengths & \multicolumn{2}{|c|}{$C=O(\AA)$} & \multicolumn{2}{|c|}{$C=S(\AA)$} \\
\hline NIS $-\mathrm{PTZ}$ & \multicolumn{2}{|c|}{$\begin{array}{l}1.2164(13) \\
1.2234(13)\end{array}$} & \multicolumn{2}{|c|}{ N/A } \\
\hline $\mathrm{NIS}_{2}-\mathrm{PTZ}$ & \multicolumn{2}{|c|}{$N / A$} & \multicolumn{2}{|c|}{$\begin{array}{l}1.644(3) \\
1.651(3)\end{array}$} \\
\hline NDIS $_{0-P T Z}$ & \multicolumn{2}{|c|}{$\begin{array}{l}1.210(4) \\
1.211(4) \\
1.214(4) \\
1.224(4)\end{array}$} & \multicolumn{2}{|c|}{$\mathrm{N} / \mathrm{A}$} \\
\hline NDIS $_{3}-\mathrm{PTZ}$ & $\begin{array}{c}\text { Molecule A } \\
1.233(6)\end{array}$ & $\begin{array}{l}\text { Molecule B } \\
1.249(6)\end{array}$ & $\begin{array}{c}\text { Molecule A } \\
1.642(5) \\
1.653(5) \\
1.653(5)\end{array}$ & $\begin{array}{c}\text { Molecule B } \\
1.638(5) \\
1.640(5) \\
1.658(5)\end{array}$ \\
\hline NDIS $_{0}$ & \multicolumn{2}{|c|}{$\begin{array}{l}1.2157(14) \\
1.2158(14) \\
1.2167(14)\end{array}$} & \multicolumn{2}{|c|}{ N/A } \\
\hline NDIS $_{1}$ & \multicolumn{2}{|c|}{$\begin{array}{l}1.185(5) \\
1.297(5)\end{array}$} & \multicolumn{2}{|c|}{$\begin{array}{l}1.645(4) \\
1.696(8)\end{array}$} \\
\hline
\end{tabular}

† for NDIS $_{3}$-PTZ two molecules are observed in the asymmetric unit. 

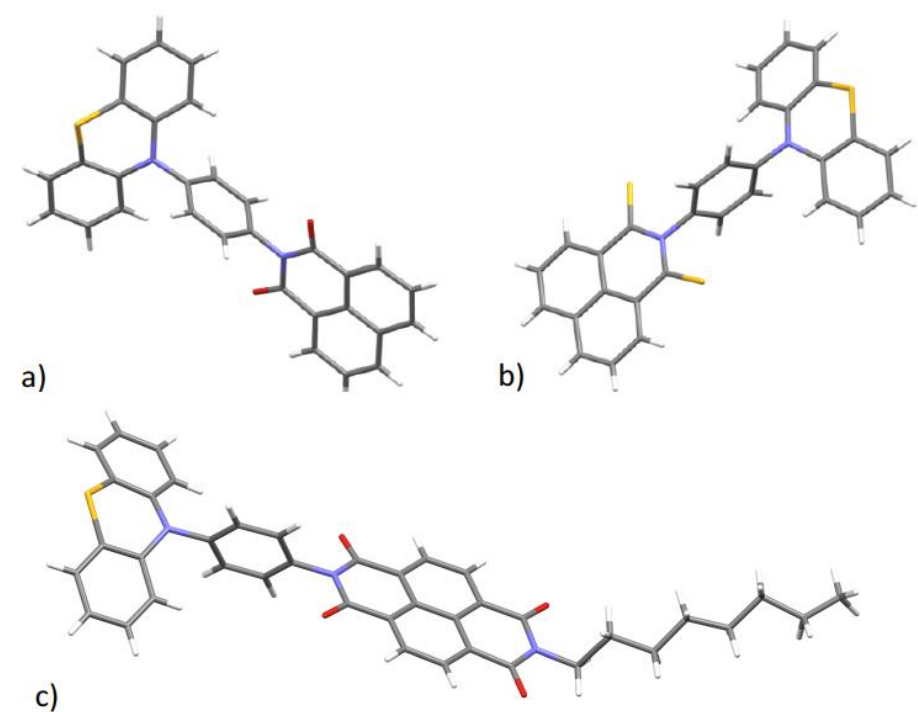

c)

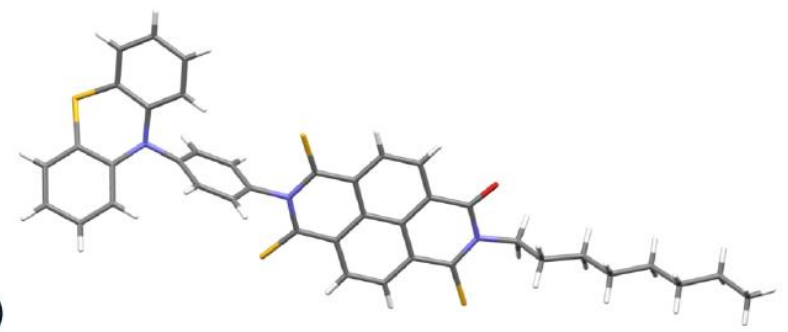

Figure 1. Views of the crystal structures of a) NIS - PTZ b) NIS - -PTZ; c) NDIS - PTZ and d) NDIS PTZ. Carbon - grey; nitrogen - blue; hydrogen - white; oxygen - red; sulfur - yellow.

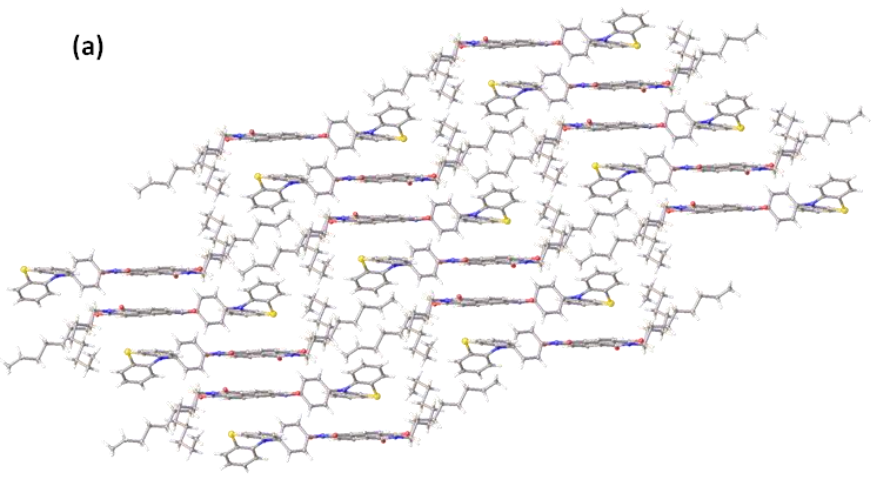

(b)

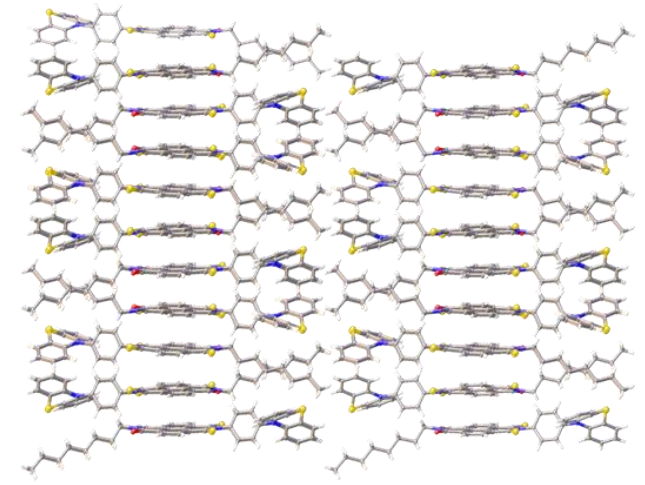

Figure 2. View of the packing arrangement of (a) NDIS - PTZ along the $b c$-axis and (b) NDIS $\mathbf{S}^{-}$ PTZ along the $a b$-axis . Carbon - grey; nitrogen - blue; hydrogen - white; and oxygen - red.

Spectroscopic, Electrochemical, Spectroelectrochemical, and EPR Measurements A clear colour change results from thionation of the NDI core. For the model thionated NDIs, the monothionated $\mathrm{NDI}$ forms a green solution in $\mathrm{CH}_{2} \mathrm{Cl}_{2}$, the dithionated NDIs form brown solutions, and the trithionated NDI forms a red solution (Figure 3). A concomitant shift occurs 
with the $\lambda_{\max }$ in the UV/visible spectra (Figure 4), representing a bathochromic shift of $\sim 45 \mathrm{~nm}$ per sulfur atom substitution, consistent with measurements of related perylene diimides. ${ }^{28-}$ 30 The highly structured absorptions of the naphthalene core change appearance upon thionation, causing the bands to broaden and move to lower energies (from $382 \mathrm{~nm}$ for NDIS to $502 \mathrm{~nm}$ for $\mathrm{NDIS}_{3}$ ). The addition of the phenothiazine chromophore in the dyads, NIS2-PTZ,

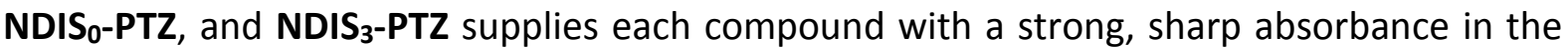
ultraviolet region at $257 \mathrm{~nm}\left(\varepsilon>40,000 \mathrm{~mol}^{-1} \mathrm{dm}^{3} \mathrm{~cm}^{-1}\right)$. Thionation of the naphthalic imide also causes a bathochromic shift to the absorption maximum. NIS $\mathbf{S}_{0}$ PTZ does not absorb any visible light and forms colourless solutions $\left(\lambda_{\max }=337 \mathrm{~nm}\right)$. The absorption maximum for $\mathbf{N I S}_{2}$ PTZ occurs at $442 \mathrm{~nm}$, representing a redshift of $105 \mathrm{~nm}$ as a result of dithionation, a more pronounced effect than the $45 \mathrm{~nm}$ shift observed in the model NDI system.

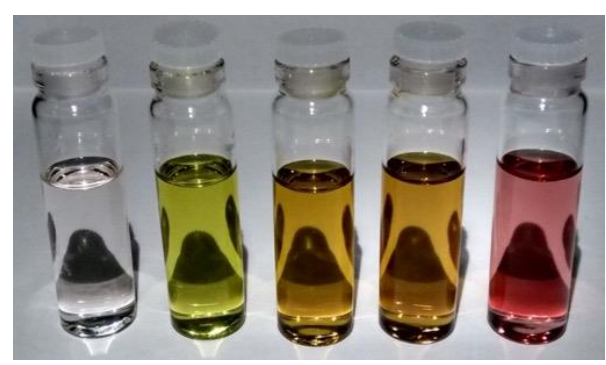

Figure 3. Solutions of thionated NDIs in dichloromethane solutions. Left to right: NDIS $\mathbf{S}_{\mathbf{0}} \mathbf{N D I S}_{\mathbf{1}_{1}}$ cis-NDIS 2 , trans-NDIS $\mathbf{2}_{2} \mathbf{N D I S}_{\mathbf{3}}$.

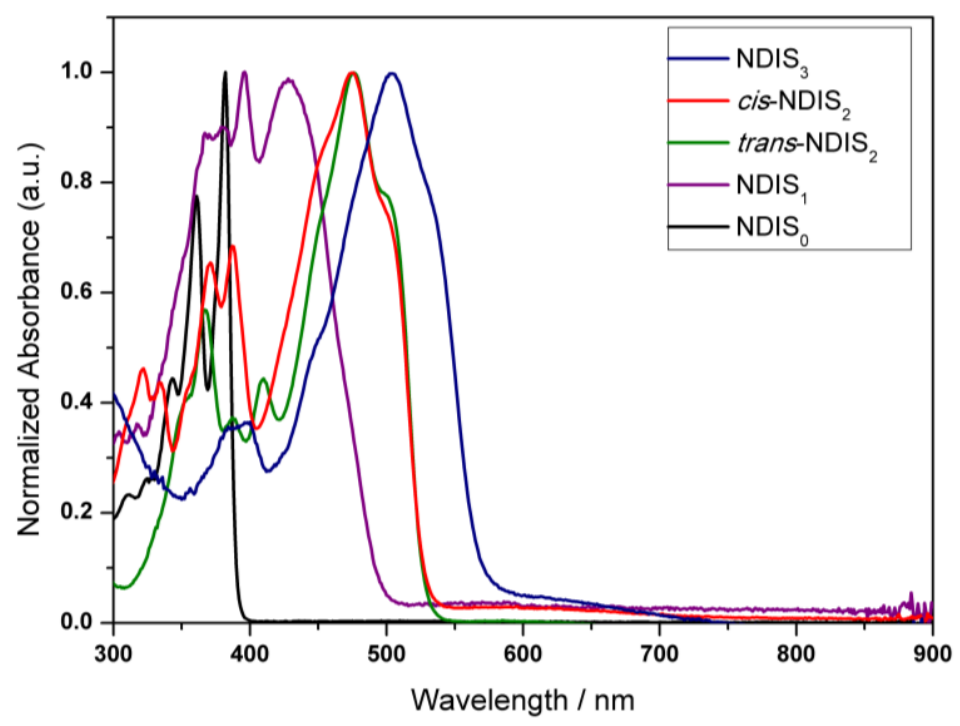

Figure 4. Normalized optical absorbance spectra of model thionated NDIs. Spectra were recorded in dichloromethane at $273 \mathrm{~K}$. 
In order to interpret the effect of thionation on the UV/visible spectra of both dyads and model compounds, time dependent (TD) calculations of the ground and triplet excited states were performed on optimised structures. Transitions that correspond to the lowestenergy bands in the UV-vis spectra are summarised with the experimental absorption data in Tables S2 and S3 and the corresponding MOs are shown in Tables S7 to S9. B3LYP basis set predicted the lowest-energy absorption maxima with a mean absolute deviation of $17 \mathrm{~nm}$. CAM-B3LYP showed a greater error, consistently over-estimating the transition energy. One exception is cis-NDIS ${ }_{2}$, for which CAM-B3LYP performed more accurately. The nature of the lowest-energy transition is in each case predicted to be $\pi \rightarrow \pi^{*}$, centred on the NI or NDI core of the molecule, confirming the occurrence of any charge-separation is a post-Franck-Condon process.

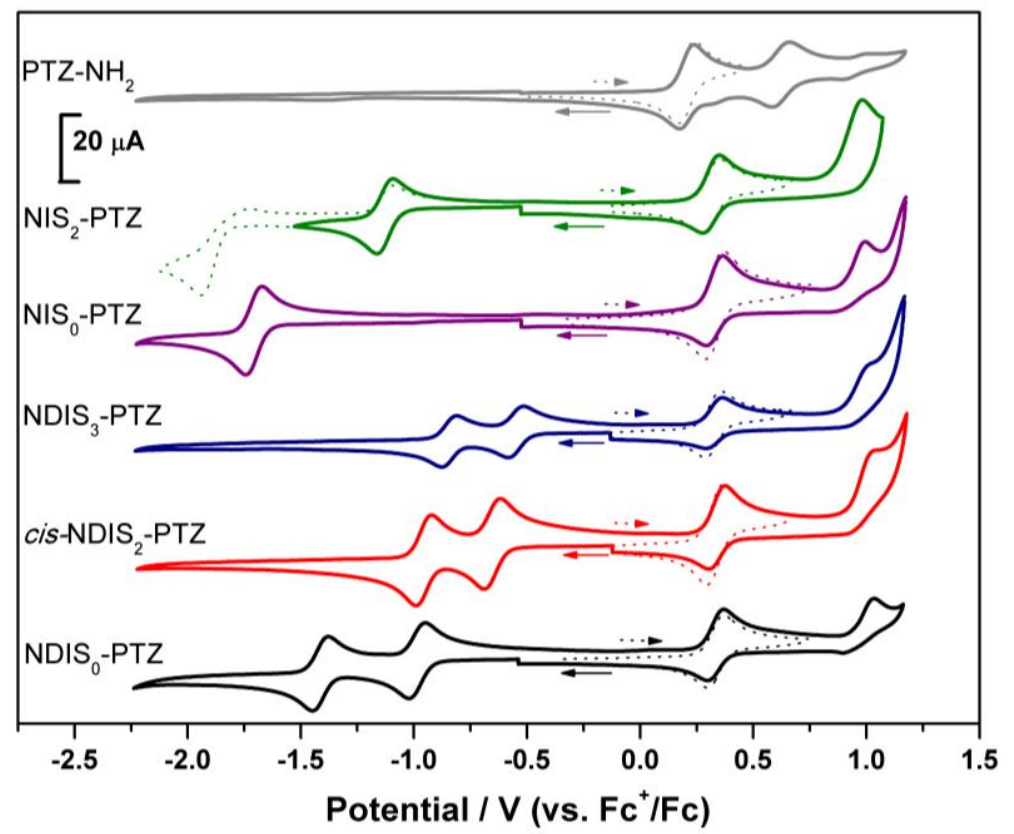

Figure 5. Cyclic voltammograms of thionated $\mathrm{NI}$ and NDI dyads in dichloromethane. Black:

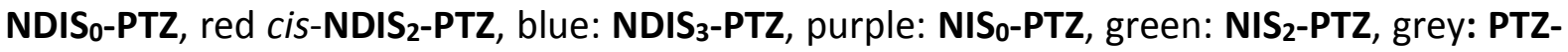
$\mathrm{NH}_{2}$.

The effect of thionation on the electrochemical behaviour of each compound was measured using cyclic voltammetry (see Figure 5 for dyads; Figure 6, for model NDI/NI compounds and Table 2). All NI dyads possessed a single reversible one-electron reduction and NDI compounds displayed two reversible one-electron reductions. Additionally, all the dyads were shown to exhibit two oxidation processes. One of which was reversible and 
resulted from the phenothiazine donor orbitals. Across all the dyads, the oxidation processes were found to occur at almost identical potentials, with a reversible oxidation at $+0.33 \mathrm{~V}$ and the irreversible oxidation at $c a .+1 \mathrm{~V}$ (no return wave was detected under the experimental conditions). Thus the thionation of the carbonyls had no effect on the phenothiazine portion of the dyads, indicating no communication between the frontier orbitals of the dyad systems. The free phenothiazine, PTZ- $\mathbf{N H}_{\mathbf{2}}$, could be oxidised more readily than the dyad systems at a lower potential of $+0.21 \mathrm{~V}$.

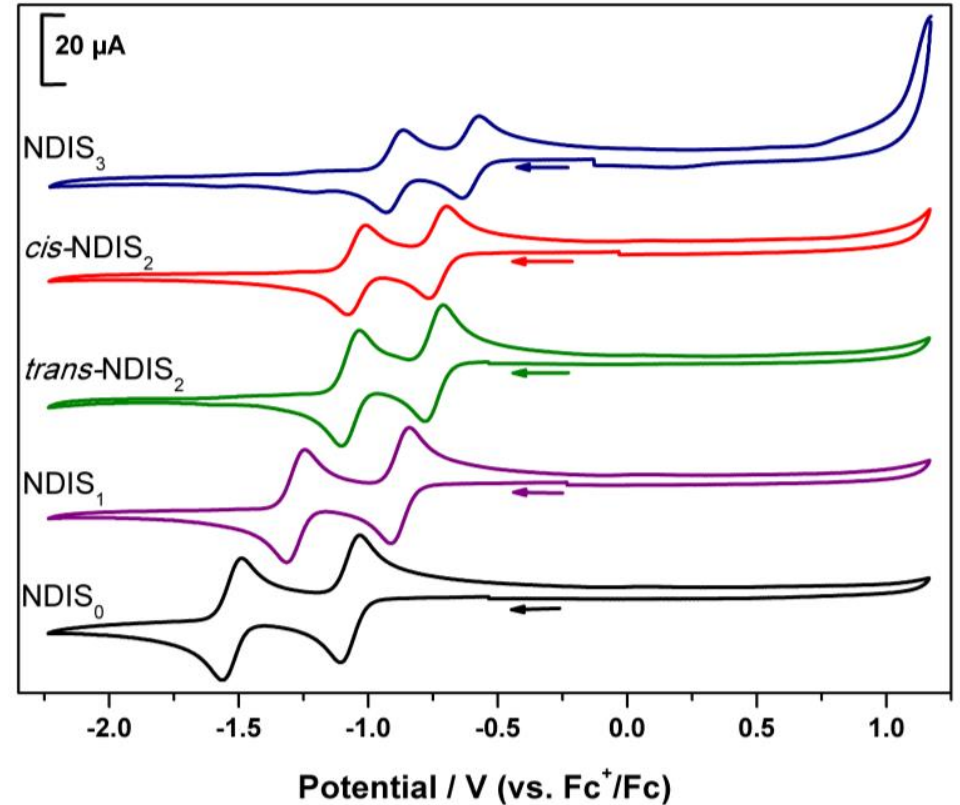

Figure 6. Cyclic voltammograms of the thionated NDI series in dichloromethane. Black: NDIS purple: $\mathbf{N D I S}_{\mathbf{1}}$, green: trans-NDIS $\mathbf{2}-\mathbf{P T Z}$, red: cis-NDIS $\mathbf{2}_{\mathbf{2}}$ blue: $\mathbf{N D I S}_{\mathbf{3}}$.

For reduction potentials upon thionation, the shift to more positive potentials is almost identical in magnitude for both the dyads and the model NDI systems (Table 2). The two reduction potentials shift anodically with thionation by $\sim 150 \mathrm{mV}$ for the first reduction and $\sim 200 \mathrm{mV}$ for the second reduction. For cis-NDIS $\mathbf{2}-\mathbf{P T Z}$, the reduction potentials are $0.36 \mathrm{~V}$ and $0.45 \mathrm{~V}$ more positive relative to its nonthionated precursor. For comparison, relative to the nonthionated molecule $\mathbf{N D I S}_{\mathbf{0}}$, the reduction potentials for cis-NDIS $\mathbf{2}$ are $0.34 \mathrm{~V}$ and 0.48 $\mathrm{V}$ more positive. The shift in redox potential is just as prominent for the dyads as for the simpler NDIs. Evaluation of the two trithionated NDIs, NDIS 3 -PTZ and NDIS $\mathbf{S}_{\mathbf{3}}$ further support this observation (NDIS - PTZ: $0.44 \mathrm{~V}, 0.56 \mathrm{~V}$; NDIS 3 : $0.46 \mathrm{~V}, 0.62 \mathrm{~V}$ ). The electron affinity of the $\mathrm{NDI}$ increases as the sulfur content of the NDI is raised, suggesting that the LUMO energy 
decreases with thionation. DFT calculations support this conclusion, with LUMO energies increasing by $150 \mathrm{meV}$ per sulfur substitution (see $\mathrm{SI}$ ). The energy of the HOMO is determined to be $c a$. $-6.21 \mathrm{eV}$ for the thionated molecules, with the orbital based primarily

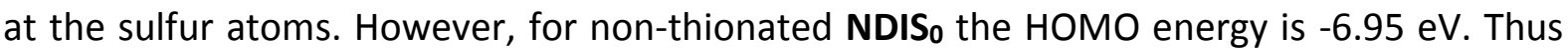
the energy difference between the LUMOs of NDIS $\mathbf{S}_{\mathbf{0}}$ and cis-NDIS $\mathbf{S}_{\mathbf{2}}$ is $0.4 \mathrm{eV}$ and similarly the difference in energy between the LUMOs of NDIS $\mathbf{S}_{\mathbf{0}}$-PTZ and cis-NDIS $\mathbf{S}_{2}$-PTZ is $0.39 \mathrm{eV}$. The trithionated NDIs exhibit similar behaviour with a difference for the model NDIs of $0.57 \mathrm{eV}$ and for the phenothiazine-NDIs of $0.56 \mathrm{eV}$; these data also suggest that the effect of thionation to the NDI system is just as prominent, regardless of imide substituents.

The most dramatic increase in reduction potential occurs for the naphthalic imide systems. NIS 2 -PTZ has a first reduction potential at $-1.13 \mathrm{~V}, 580 \mathrm{mV}$ higher than that of its non-thionated precursor, NIS $\mathbf{S}_{0}$-PTZ. Typical NDIs such as NDIS $\mathbf{S}_{0}$ have first reduction potentials of $\sim-1.1 \mathrm{~V}$, hence the effect of full sulfur substitution of the naphthalic imide molecule influences the electron affinity as significantly as the addition of a second imide group to form an NDI (though it does not add a second reduction process). This shift in reduction potential is particularly significant since NIs are more synthetically accessible than NDIs and lend themselves to further functionalization.

Table 2. Reduction potentials of a thionated NDI compound series.

\begin{tabular}{|c|c|c|c|c|}
\hline Compound & $\begin{array}{l}1^{\text {st }} \text { Reduction } \\
E_{1 / 2} / V\end{array}$ & $\begin{array}{l}2^{\text {nd }} \text { Reduction } \\
E_{1 / 2} / V\end{array}$ & $\begin{array}{l}\text { 1st Oxidation } \\
E_{1 / 2} / V\end{array}$ & $\begin{array}{l}\text { 2nd Oxidation } \\
E_{p a} / V\end{array}$ \\
\hline NDIS $_{0}-\mathrm{PTZ}$ & -0.99 & -1.41 & 0.33 & 0.98 \\
\hline cis-NDIS 2 -PTZ & -0.63 & -0.96 & 0.33 & 1.02 \\
\hline $\mathrm{NDIS}_{3}-\mathrm{PTZ}$ & -0.55 & -0.85 & 0.32 & 1.00 \\
\hline NIS $_{0}-\mathrm{PTZ}$ & -1.71 & & 0.33 & 0.99 \\
\hline NIS $_{2}-\mathrm{PTZ}$ & -1.13 & & 0.32 & 0.99 \\
\hline PTZ-NH & & & 0.21 & $0.63 *$ \\
\hline NDIS $_{0}$ & -1.07 & -1.53 & & \\
\hline NDIS $_{1}$ & -0.88 & -1.29 & & \\
\hline trans-NDIS 2 & -0.75 & -1.08 & & \\
\hline cis-NDIS $\mathbf{2}$ & -0.73 & -1.05 & & \\
\hline $\mathrm{NDIS}_{3}$ & -0.61 & -0.91 & & \\
\hline NIS & -1.82 & & & \\
\hline $\mathrm{NIS}_{1}$ & -1.44 & & & \\
\hline
\end{tabular}

Potentials quoted against $\mathrm{E}_{1 / 2} \mathrm{Fc}^{+} / \mathrm{Fc}$ at $100 \mathrm{mVs}^{-1}$ used as the internal standard. Recorded in dichloromethane at ambient temperature containing $\left[{ }^{n} \mathrm{Bu}_{4} \mathrm{~N}\right]\left[\mathrm{BF}_{4}\right](0.4 \mathrm{M})$ as the supporting electrolyte. ${ }^{*} \mathrm{E}_{1 / 2}$ value given. 
Spectral profiles for the various oxidation states of the compounds were generated using spectroelectrochemical techniques (the optical absorbance spectra recorded for the singly-oxidised (dyads only), singly-reduced and double-reduced states of the dyads) (see Figure 7 for representative example of cis-NDIS2-PTZ and Figure 8 NDI series). Oxidation of the dyads for NDISo-PTZ and NIS $\mathbf{S}_{0}$-PTZ led to very little perturbation of the absorption bands between 300 and $400 \mathrm{~nm}$ (attributed to the naphthalene-imide rings of the donors). Changes

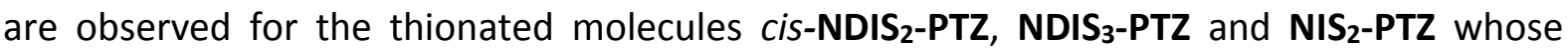
absorptions are red-shifted close to the $500 \mathrm{~nm}$, and thus overlap with the emerging band at $515 \mathrm{~nm}$ from the oxidised phenothiazine. The strong absorbance in the UV at $257 \mathrm{~nm}$ shifts to $276 \mathrm{~nm}$ upon oxidation. A lack of communication between the chromophores reinforces that separation of the frontier orbitals has occurred, indicating that the dyads are viable candidates for charge transfer systems.

Reduction of the NDI-PTZ dyads proceeds in an analogous manner to model NDI species with spectra for the monoanionic and dianionic NDI-PTZ compounds closely resembling those of the corresponding model NDIs. The NDI frontier orbitals, and hence the chromophore, are unaffected by functionalisation at the imide nitrogen atoms and substitutions to these positions are not anticipated to affect the overall profile. Each monoanionic NDI exhibits a similar absorption profile, with a small variation observed for trans-NDIS2. Reduction to the dianionic species generated chromophores with wellstructured bands at higher energy and an additional set of bands with lower intensities at lower energy wavelengths. Trans-NDIS $\mathbf{2}$ is an exception with more intense lower energy bands. Thionation of NDIS $\mathbf{S}_{0}$-PTZ caused a redshift of the NDI bands, which was preserved upon reduction. The absorption spectra for the various redox states of cis-NDIS 2 -PTZ (Figure 7) proved to be a useful tool for the characterisation of the compound. The spectrum for the monoanionic form of cis-NDIS $\mathbf{2}$-PTZ strongly resembled that of cis-NDIS 2 , with one intense peak at $\lambda_{\max }=596 \mathrm{~nm}$. Reduction of the two NI-PTZ compounds saw the emergence of new, lower energy bands at $419 \mathrm{~nm}$ for NIS $\mathbf{S}_{0}$-PTZ and $458 \mathrm{~nm}$ for NIS $_{2}$-PTZ. Whilst this occurred for the related NDI compounds, the effect of the thionation is significantly diminished for the NIs. The most intense absorption band for the monoanionic form of NIS $_{2}-\mathbf{P T Z}$ is shifted by a mere $16 \mathrm{~nm}$ relative to its neutral counterpart, despite the nonthionated $\mathbf{N I S}_{\mathbf{0}}$-PTZ displaying a sharp band redshifted by $82 \mathrm{~nm}$. Additionally, lower intensity absorptions $\left(\varepsilon>10,000 \mathrm{~mol}^{-}\right.$ 
${ }^{1} \mathrm{dm}^{3} \mathrm{~cm}^{-1}$ ) developed in the near infrared region at 833 and $748 \mathrm{~nm}$ for both NI dyads (see Table S3).
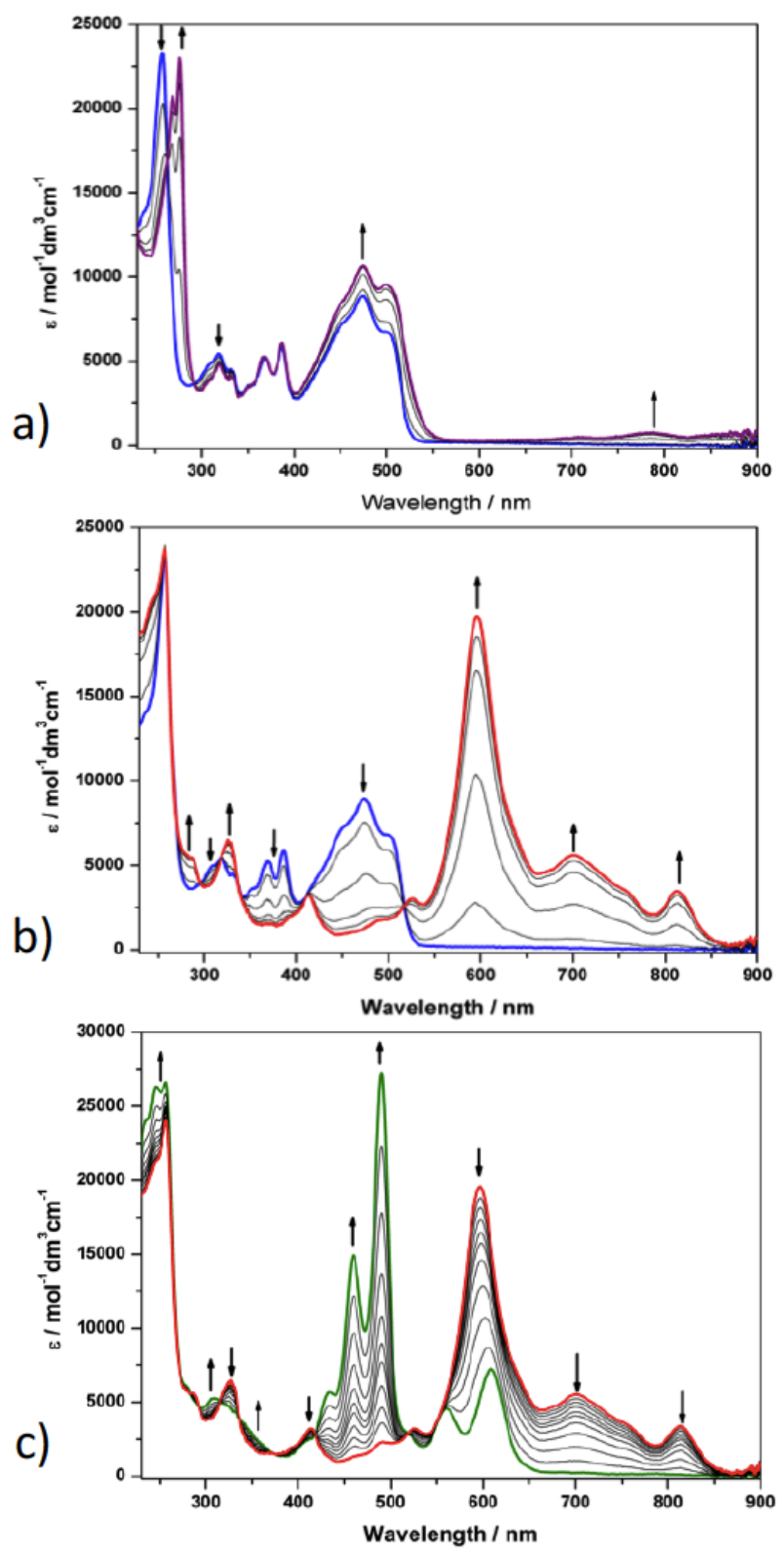

Figure 7. UV-vis absorption spectra showing the inter-conversion between the various charge states of cis-NDIS - -PTZ. Arrows indicate the progress of the oxidation/reduction a) from neutral (blue) to monocationic (purple), b) from neutral (blue) to monoanionic (red), c) from monoanionic (red to dianionic (green) species. Spectra were recorded in dichloromethane containing $\left[{ }^{\mathrm{n} B \mathrm{H}_{4} \mathrm{~N}}\right]\left[\mathrm{BF}_{4}\right](0.4 \mathrm{M})$ as the supporting electrolyte at $273 \mathrm{~K}$. 

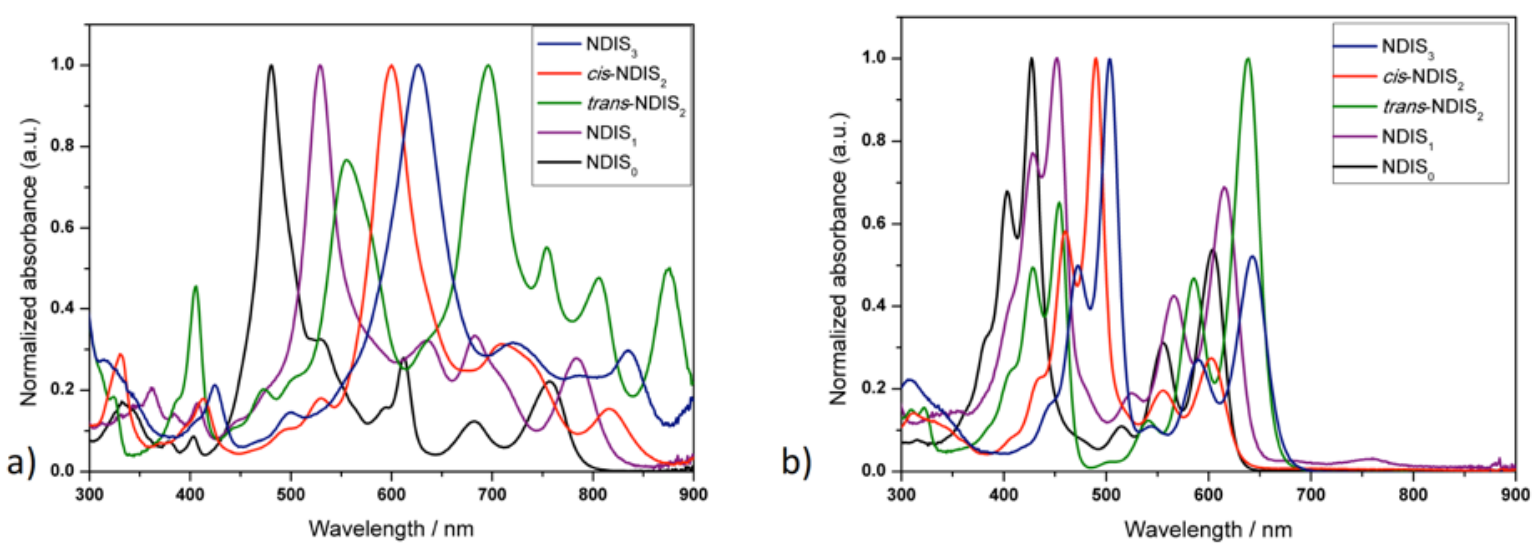

Figure 8. Optical absorbance spectra of a) monoanionic thionated NDIs and b) dianionic thionated NDIs. Spectra were recorded in dichloromethane containing [ $\left.{ }^{n} \mathrm{Bu}_{4} \mathrm{~N}\right]\left[\mathrm{BF}_{4}\right](0.4 \mathrm{M})$ as the supporting electrolyte at $273 \mathrm{~K}$.

Electron paramagnetic resonance (EPR) experiments were conducted to determine any differences in localisation of the radical electron in the oxidised monocationic and reduced monoanionic forms of the dyad molecules. NIs and NDIs are known to function as electron acceptors, so one would expect that reduction of these compounds would be achieved through the addition of an electron into a LUMO orbital located on the NI or NDI unit. Similarly, with phenothiazine as an electron donor, oxidation should cause the loss of an electron from a HOMO orbital located on the phenothiazine chromophore. The EPR data obtained agrees with this hypothesis. Distinct spectra are produced for oxidised and reduced forms of the same molecule, indicating that the locations of the radical electrons for the monocationic and monoanionic species are distinct and based upon the phenothiazine and $\mathrm{NDI} / \mathrm{NI}$ chromophores respectively. Moreover, a change in the acceptor between NDI and NI (as well as thionation) affects the EPR spectrum of the reduced compound. Since all dyads contain the same PTZ electron donor, the EPR spectra for the oxidized molecules are largely consistent (Figure 9a). From the similarity of the EPR spectra it can be readily deduced that the HOMO of all the dyads lies on the PTZ portion of the molecule. Furthermore, experimentally determined $g_{\text {iso }}$ values are identical for all oxidised dyads at 2.0055 and only marginally different for PTZ-NH $\mathbf{N H}_{2}$ at 2.0053. DFT calculated molecular orbitals for the structures further support this conclusion (SI).

EPR spectra of the radical anions of the thionated NDI-PTZ and NDI series generated by bulk electrolysis are consistent with the radical electron confined to a $\pi$-orbital based around the aromatic NDI core. Hyperfine coupling was displayed between the radical electron 
and the hydrogen atoms of the aromatic core and the imide nitrogens. The DFT generated orbital diagrams (see SI) for the thionated NDIs are in agreement with this experimentally obtained data. The LUMOs for all the NDIs have similar structure, with molecular orbitals predominantly based on sulfur atoms.

a)
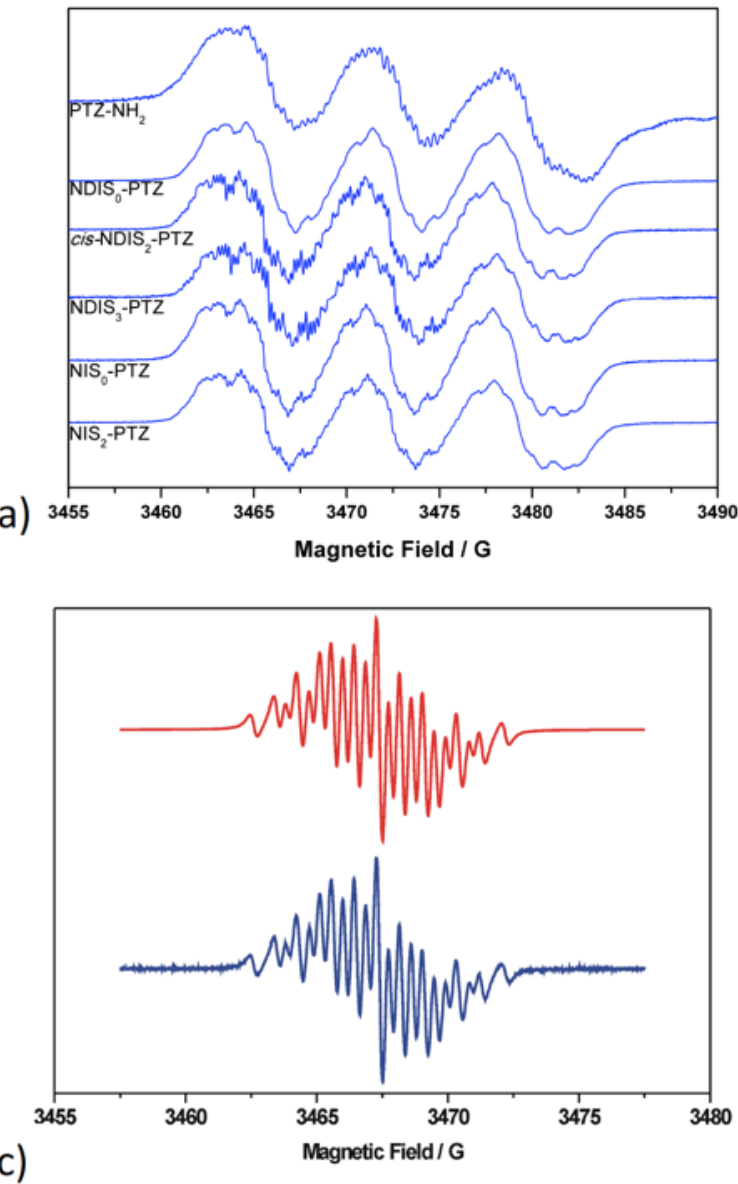

b)
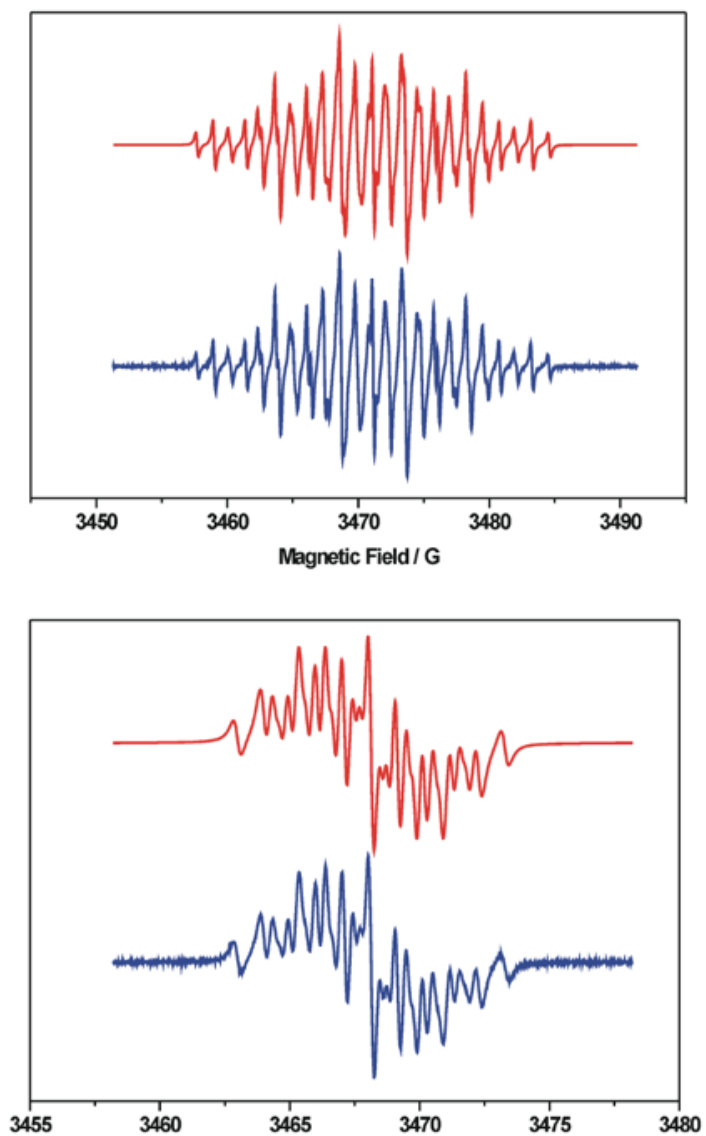

d)

Figure 9. a) Experimentally obtained EPR spectra of oxidised NDI-PTZ and NI-PTZ dyads; EPR spectra of reduced b) NDIS $-\mathbf{P T Z}$; c) cis-NDIS $\mathbf{S}_{\mathbf{2}}$ and d) trans-NDIS $\mathbf{2}_{2}$. simulated. Blue: experimental, red: simulated.

The EPR spectrum of [NDISo-PTZ] showed more complexity than the simpler

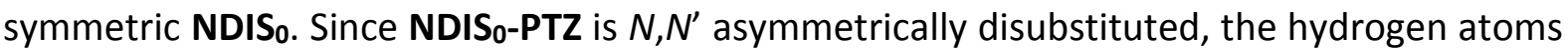
located on the aromatic core of the NDI exist in two pairs of equivalent environments, leading to increased complexity of the spectrum, instead of all four hydrogens being symmetrically equivalent in a symmetrically substituted NDI. Simulation of the experimental data to obtain values for hyperfine coupling constants was possible (Figure 9b), although also required coupling to an additional set of two equivalent protons, revealing that a weak coupling of the 
radical electron to the imide-alkyl chain is also present. The general shape of thirteen lines (the case for NDIS ) is present, although each of these is split further by the extra interactions. Extension of the delocalization of the radical electron into the first carbon of the imide position is occasionally observed for hydrocarbon chains. ${ }^{5,32}$ The two imide nitrogen atoms lie in chemically distinct environments; one bears an alkyl substitution, the other aryl, though a difference in their hyperfine contribution to the EPR spectrum is negligible and simulation of the recorded data did not require the fitting of two independent nitrogen parameters. Despite the differences in spectra, both non-thionated NDI radicals have virtually identical $g_{\text {iso }}$ values at 2.0037 for NDIS ${ }_{0}$ and 2.0038 for NDIS $_{0}-$ PTZ. The $g_{\text {iso }}$ value of cis-NDIS - PTZ was found to be 2.0085 , identical to that recorded for cis-NDIS 2 , reinforcing the structural assignment as cis, since the trans isomer has a lower $g_{\text {iso }}$ value of 2.0078 .

Table 3. Experimentally determined $g_{\text {iso }}$ values for radical species.

\begin{tabular}{|c|c|c|c|c|c|}
\hline $\begin{array}{c}\text { Compoun } \\
\text { d }\end{array}$ & $\begin{array}{c}g_{\text {iso }} \\
\text { Mono- } \\
\text { reduce } \\
\text { d }\end{array}$ & $\begin{array}{c}g_{\text {iso }} \\
\text { Mono- } \\
\text { oxidise } \\
\text { d }\end{array}$ & Compound & $\begin{array}{c}g_{\text {iso }} \\
\text { Mono- } \\
\text { reduce } \\
\text { d }\end{array}$ & $\begin{array}{c}g_{\text {iso }} \\
\text { Mono- } \\
\text { oxidise } \\
\text { d }\end{array}$ \\
\hline NDIS $_{0}-\mathrm{PTZ}$ & 2.0038 & 2.0055 & cis-NDIS $-\mathrm{PTZ}$ & 2.0085 & 2.0055 \\
\hline NDIS $_{3}-\mathrm{PTZ}$ & 2.0110 & 2.0055 & $\mathrm{NIS}_{0}-\mathrm{PTZ}$ & 2.0032 & 2.0055 \\
\hline $\mathrm{NIS}_{2}-\mathrm{PTZ}$ & 2.0062 & 2.0055 & & & \\
\hline PTZ-NH 2 & & 2.0053 & NDIS $_{0}$ & 2.0037 & \\
\hline NDIS $_{1}$ & 2.0058 & & trans-NDIS $\mathbf{2}$ & 2.0078 & \\
\hline cis-NDIS & 2.0085 & & NDIS $_{3}$ & 2.0105 & \\
\hline $\mathrm{NIS}_{0}$ & 2.0032 & & $\mathbf{N I S}_{1}$ & 2.0046 & \\
\hline
\end{tabular}

EPR spectra recorded for the dithionated NDIs, cis-NDIS $\mathbf{S}_{\mathbf{2}}$ and trans-NDIS $\mathbf{2}$ (Figure 9c,d) display some differences, confirming that the symmetry of the aromatic core affects the observed hyperfine coupling. A general trend is observed in the $g_{\text {iso }}$ values of the series (Table 3), with an incremental increase of 0.0023 per additional sulfur atom. This shift, that results from progressively larger $\pi$-spin population across the series, was due to an increase in spinorbit coupling, an effect promoted by the presence of increasing numbers of heavier sulfur atoms. Simulation of the EPR data for the two NI dyads was possible (see SI), with three pairs of hydrogen atoms and one nitrogen atom contributing hyperfine coupling to the overall shape of the spectra. Significantly more coupling to the nitrogen atom is observed for the

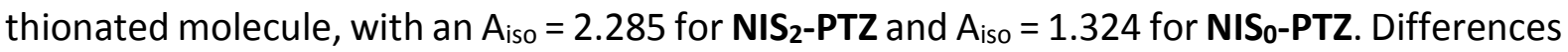
in the hydrogen hyperfine coupling constants are also apparent with larger values for the nonthionated compound. DFT computed orbital structures indicate a larger contribution from the 
thiocarbonyl carbons to the LUMO of $\mathbf{N I S}_{2}-\mathbf{P T Z}$, than on the carbonyl carbon atoms of NIS $\mathbf{S}^{-}$ PTZ with nodes at carbons 3 and 6 on the naphthyl ring (see SI).

\section{Transient Spectroscopy}

In order to evaluate the existence of charge separated states TA and TRIR spectra were recorded for both dyads and model compounds. The band positions and lifetimes of all compounds are summarised in Table 4. Furthermore, TRIR spectra of the triplet state have been simulated by subtracting the unscaled calculated ground state from the calculated triplet state spectra (see Figure S12). As expected, the energies of the (unscaled) calculated vibrations are over-estimated with respect to experiment. However, a good qualitative agreement is observed between the simulated and final states in the TRIR spectra.

To aid discussion the behaviour of the model compounds will be covered first. The

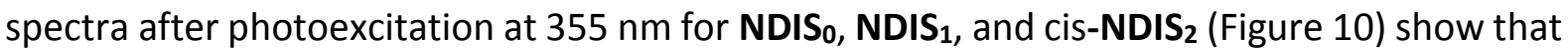
each compound possesses spectral bands that correspond to two or more potential states. As previously reported, ${ }^{33-37}$ photoexcitation of NDIS $\mathbf{S}_{0}$ at $355 \mathrm{~nm}$ leads to the appearance of two bands in the visible region, centred at 465 and $610 \mathrm{~nm}$ with a shoulder at $574 \mathrm{~nm}$. These bands decay over the first few picoseconds to produce new absorptions at 460 and $490 \mathrm{~nm}$, characteristic of a triplet $\pi \rightarrow \pi^{*}$ state. In our experiments, global fitting showed that the 460 and $490 \mathrm{~nm}$ bands have a further decay component with a lifetime of 160 ( \pm 60$)$ ps which may possibly indicate aggregation. The TRIR spectra of NDIS $_{0}$ show a depletion of ground-state bands and the appearance of new bands shifted to lower energies. Consistent with the TA data above, the initially formed bands decay to form final peaks at 1642 and $1605 \mathrm{~cm}^{-1}$, which remain until the end of the ps experiment and are consistent with bands at 1647 and 1607 $\mathrm{cm}^{-1}$ previously assigned as the triplet excited state of NDI covalently linked in a Pt complex. ${ }^{36}$ The final excited state of NDIS possesses a lifetime of $11( \pm 1) \mu \mathrm{s}$, although this is highly concentration dependent, indicating the presence of self-quenching interactions.

The introduction of sulfur into the NDI framework is expected to affect the photophysics of the species studied. The TA and TRIR spectra of NDIS 1 are representative of the other model compounds, cis-NDIS $\mathbf{S}_{\mathbf{2}}$ and trans-NDIS $\mathbf{S}_{\mathbf{2}}$, and are summarised in Table 4. The TA spectrum of NDIS ${ }_{1}$ shows a band at $561 \mathrm{~nm}$ with a shoulder at $608 \mathrm{~nm}$ which decay rapidly (4 ( \pm 3 ) ps), which corresponds to growth of bands at ca. 475 and $640 \mathrm{~nm}$ (intensity is also 
retained at $574 \mathrm{~nm}$ ) that decays with a lifetime of $490( \pm 80) \mathrm{ps}^{+}$The TRIR spectrum shows major bands at 1675,1654 , and $1632 \mathrm{~cm}^{-1}$ at early times following excitation and over the first 9 ( \pm 6$)$ ps an increase in intensity is observed at 1704, 1675, and $1578 \mathrm{~cm}^{-1}$ (corresponding to a parent recovery), while the 1652 and $1624 \mathrm{~cm}^{-1}$ regions of the spectra diminish in intensity. The remaining bands can be fitted to decay in $530( \pm 30)$ ps, at the same time as parent recovery. This is consistent with the excited-state lifetimes obtained from the ns-TRIR experiment as well as TA. The TA spectra of cis-NDIS $\mathbf{2}$ and trans-NDIS $\mathbf{S}_{\mathbf{2}}$ show similar behaviour to NDIS ${ }_{1}$ (see Figure S13 for the TA and TRIR spectra of trans-NDIS $\mathbf{S}_{2}$ ). Analogously to NDIS the early-time process in the spectra of NDIS $_{\mathbf{1}}$ and cis/trans-NDIS $\mathbf{2}$ are assigned to a singlet to triplet transition.

Table 4. Summary of the excited states found for each compound by global analyses of the visible TA and TRIR data. All measurements were carried out in $\mathrm{CH}_{2} \mathrm{Cl}_{2}$ after photoexcitation at $355 \mathrm{~nm}$.

\begin{tabular}{|c|c|c|c|c|}
\hline & $\lambda_{T A} / \mathrm{nm}$ & $\tau / \mathrm{ps}$ & $V_{T R I R} / \mathrm{cm}^{-1}$ & $\tau / \mathrm{ps}$ \\
\hline \multirow[t]{3}{*}{ NDIS $_{0}$} & 472,612 & $1( \pm 1)$ & $1725,1696,1656,1600,1576,1556$ & $2( \pm 1)$ \\
\hline & 465 & $9( \pm 5)$ & $1705,1665,1608,1588$ & $11( \pm 8)$ \\
\hline & 463 & $160( \pm 60)$ & $1704,1662,1612,1590$ & $200( \pm 90), 1.1( \pm 0.1) \times 10^{7 a}$ \\
\hline \multirow[t]{2}{*}{ NDIS $_{1}$} & 561,605 & $4( \pm 3)$ & 1652,1624 & $9( \pm 6)$ \\
\hline & $472,540,577,625$ & $490( \pm 80)$ & $1675,1654,1633$ & $530( \pm 30)$ \\
\hline \multirow[t]{2}{*}{ cis-NDIS ${ }_{2}$} & $508,553,614,677$ & $9( \pm 3)$ & 1636 & $10( \pm 5)$ \\
\hline & 476,542 & $2500( \pm 500)$ & 1657 & $2300( \pm 100)$ \\
\hline \multirow[t]{2}{*}{ trans-NDIS ${ }_{2}$} & $494,562,608$ & $3( \pm 3)$ & 1630 & $10( \pm 5)$ \\
\hline & $548,586,639$ & $2000( \pm 1000)$ & 1645 & $2200( \pm 400)$ \\
\hline \multirow[t]{3}{*}{ NDIS - PTZ } & 588,607 & $0.7( \pm 0.5)$ & $1705,1667,1566$ & $1( \pm 1)$ \\
\hline & $487,541,621$ & $7( \pm 1)$ & $1627,1593,1512$ & $9( \pm 3)$ \\
\hline & $485,534,615$ & $130( \pm 60)$ & $1630,1594,1516,1506$ & $120( \pm 30), 2( \pm 0.8) \times 10^{5}$ \\
\hline \multirow[t]{3}{*}{$\mathrm{NDIS}_{3}-\mathrm{PTZ}$} & 573,648 & $9( \pm 4)$ & 1622,1497 & $8( \pm 4)$ \\
\hline & 585 sh, 616 & $300( \pm 100)$ & $1629,1594,1514$ & $200( \pm 100)$ \\
\hline & 542sh, 569sh, 614 & $2000( \pm 2000)$ & $1654,1643,1497$ & $3000( \pm 2000)$ \\
\hline \multirow[t]{3}{*}{$\mathrm{NIS}_{0}-\mathrm{PTZ}$} & 490,603 & $1( \pm 1)$ & $1679,1612,1584,1546$ & $2( \pm 1)$ \\
\hline & 608 & $23( \pm 8)$ & 1636,1619 & $35( \pm 10)$ \\
\hline & $507 s h, 524$ & $260( \pm 60)$ & $1627,1561,1527,1501$ & $250( \pm 30)$ \\
\hline \multirow[t]{4}{*}{$\mathrm{NIS}_{2}-\mathrm{PTZ}$} & $452,517,586$ & $1.3( \pm 0.5)$ & $1571,1520,1507$ & $3( \pm 2)$ \\
\hline & 505,560 & $30( \pm 10)$ & 1566 & $30( \pm 10)$ \\
\hline & 496,522 & $2000( \pm 1000)$ & $1598,1577,1569,1495$ & $1000( \pm 100)$ \\
\hline & & & $1535,1519,1498$ & $5000( \pm 1000)$ \\
\hline
\end{tabular}

${ }^{a}$ At $2.3 \times 10^{-3} \mathrm{molL}^{-1}$; this value is highly concentration dependent.

+ Global analysis of the TA spectrum of NDIS 1 reveals that the second component possesses bands at 473,576 and $625 \mathrm{~nm}$. 


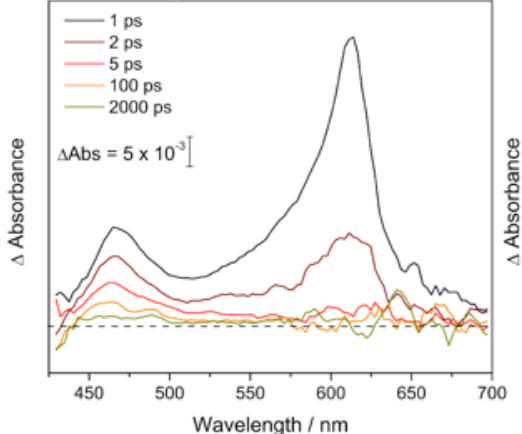

(a)

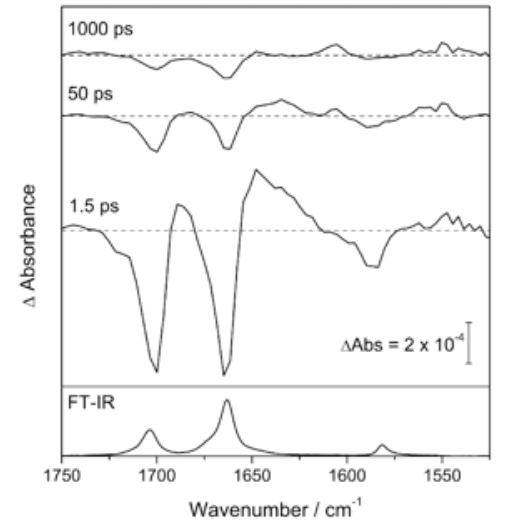

(d)

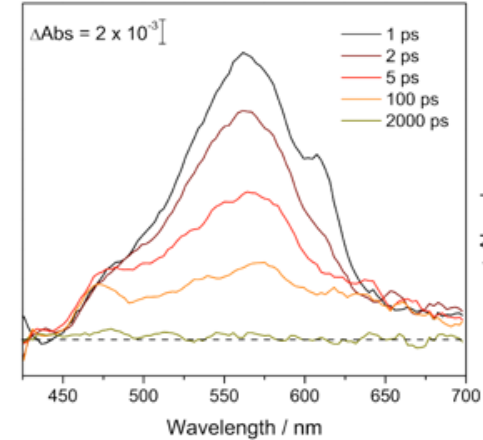

(b)

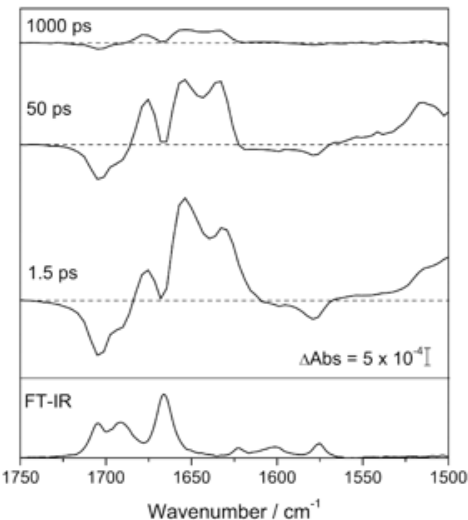

(e)

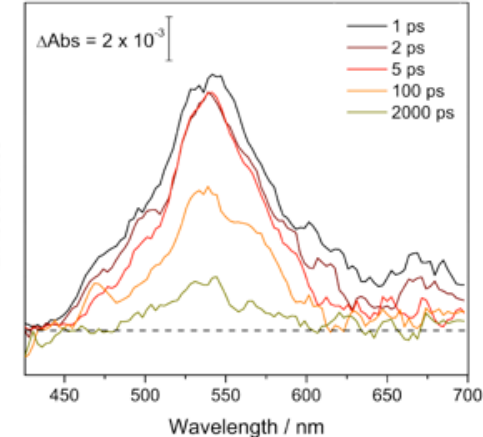

(c)

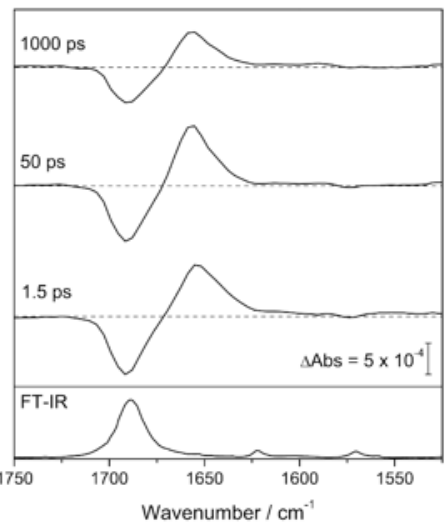

(f)

Figure 10. (a)-(c) Transient absorption and (d)-(f) TRIR spectra acquired in $\mathrm{CH}_{2} \mathrm{Cl}_{2}$ at selected time delays after photoexcitation with a $355 \mathrm{~nm}$ pulse. In (d)-(f) the corresponding FT-IR spectra are also shown; (a, d) NDIS,$(b, e) \mathbf{N D I S}_{1}$, and (c, f) cis-NDIS .

The photophysical behaviour of the compounds $\operatorname{NDIS}_{\mathbf{n}}(\mathrm{n}=0,1,2)$ can be considered as a group. Initially singlet excited states are formed, which convert rapidly to triplet states before decaying to the ground state. It is clear that the presence of the sulfur substituents decreases the excited state lifetimes that are reduced by four orders of magnitude compared to NDIS. A potential origin for this is the increased spin-orbit coupling for the sulfurcontaining compounds and the excited states of these show a large degree of sulfur-character as evident from the triplet MOs as well as the HOMOs (Table S7, SI). This may facilitate a more efficient return to the singlet ground state in the thionated compounds and may also mean the nature of the excited state has changed. The calculated lowest energy triplet states for the model compounds can be described as $\pi \rightarrow \pi^{*}$, consisting of a donor and acceptor that are both delocalised over the majority of the NDI core. The energy gap between the calculated singlet and triplet states can act as a guide for the triplet excited state energy. It is calculated 
as ca. $1.98,1.55,1.31$, and $1.45 \mathrm{eV}$ for NDIS$_{0}$, NDIS$_{1}$, cis-NDIS $\mathbf{2}_{2}$, and trans-NDIS 2 respectively, showing a decrease with increasing number of sulfur substituents and following a similar trend as the calculated HOMO-LUMO gap of the singlet state (Figure S9 and Table S10) as well as the reduction potentials (Table 2 ).

The measurements above show that excited state behaviour is greatly affected by thionation of the NDI. Thus, we examined charge separation in the dyads NDISo-PTZ, NDIS PTZ, NIS 0 -PTZ and NIS - PTZ. DFT calculations confirm that the ground state HOMOs of the PTZ containing dyads are located on the PTZ subunits, indicating that any charge separated excited singlet states are likely of lower energies than the initially formed $\pi \rightarrow \pi^{*}$ states. Similarly, the frontier MOs of the triplet states are indicative of where the electron and hole are located in the lowest energy excited state: the location of the excited state electron is calculated as the NI or NDI core; however, the hole is clearly situated on the PTZ moiety. Therefore, charge separation is expected to take place in the presence of a PTZ donor. For all compounds, the PTZ units appear to be planar in the triplet state, which is in contrast to the bowed geometries of the ground state geometries, see calculations and single crystal X-ray structures above. Conversely, the phenyl linker remains almost completely normal to the planes of the PTZ and NDI/NI units in the calculated triplet state, indicating that there is little to no change in the linking unit.

The TA and TRIR spectra of NDIS - -PTZ, NIS - -PTZ and NIS $_{2}-\mathbf{P T Z}$ are shown in Figure 11.

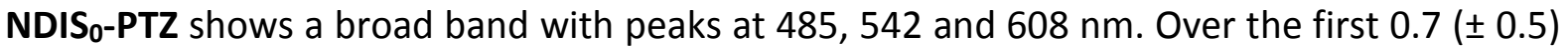
ps the band at $608 \mathrm{~nm}$ decreases in intensity, concurrent with a growth in the $500 \mathrm{~nm}$ region. The final bands, at 485, 535 and $615 \mathrm{~nm}$, decay biexponentially $(7( \pm 1)$ ps and $130( \pm 60)$ ps) and a small residual is retained at the end of the picosecond experiment. The results of spectroelectrochemical measurements, discussed above, were used to aid assignment of the final spectrum. The electrochemically oxidised species for all compounds containing the PTZ donor show marker bands for PTZ. ${ }^{+}$at $520 \mathrm{~nm}$, as has been reported previously ${ }^{38-40}$ whilst the electrochemically reduced NDI-containing species shows significant bands at 478 and $609 \mathrm{~nm}$. Thus, the TA spectra of NDIS - PTZ appear similar to a superposition of the electrochemically reduced and oxidised UV-vis spectra (Figure 11). Furthermore, the spectrum of the reduced species strongly resembles that of reduced NDIS $_{0}$ (see above), indicating that reduction leads to electronic population of the NDI core of the molecule. 
The TRIR spectra of NDIS $\mathbf{S}_{0}$-PTZ show depletions of the parent bands where expected from the FT-IR spectra and transient bands shifted to lower wavenumbers. A fast initial process $(1( \pm 0.5)$ ps) leads to an intensification of the transient bands with little change in the parent bands. Similar to the TA data, two exponentials are required to fit the decay of the picosecond spectra and the fast ( 9 ps) process is accompanied by a recovery at the position of the parent depletions. Little change in the spectral shape occurs, although the final bands appear to be shifted to slightly higher energies. The final TRIR spectra clearly show features of the IR spectrum of the electrochemically reduced species (see Figure S9), supporting the presence of a charge-transfer state. A residual persists to the end of the picosecond experiment, which was further probed using ns-TRIR. This experiment found a relatively long excited state lifetime of $200( \pm 80)$ ns; little change in the transient spectra from the picosecond timescale to the final decay was observed. The superposition in the UV and IR absorption spectra of the donor and acceptor species strongly suggest the excited state to be of charge-transfer character and there is precedence for charge-transfer from previously examined NDI assemblies. ${ }^{41-43}$

The initial fast process may be due to a charge-separation process. This is consistent with singlet-state TD-DFT calculations, which predict an initial excitation solely based on the NDI subunit with charge-separation occurring at a later time. However, this is not definitive and other singlet to triplet processes may be occurring. The initial increase in intensity of the transient bands indicates that the first state formed possesses a weak infrared absorption and is therefore not very diagnostic of the nature of the state.

Both the TA and TRIR spectra of NDIS $_{3}$-PTZ (see Figure S14, SI) require three components to fit the kinetics satisfactorily (Table 4). The TA spectrum shows a broad band at ca. $600 \mathrm{~nm}$ with a depletion at $500 \mathrm{~nm}$, corresponding to the ground state absorption spectrum. The initial $9( \pm 4)$ ps component shows the depletion of bands at 573 and $647 \mathrm{~nm}$, overlapping with an increase in intensity at $622 \mathrm{~nm}$; the latter matches well with the spectroelectrochemical measurements of the UV-visible spectrum of the reduced species. An increase in intensity observed at $500 \mathrm{~nm}$ matches with the spectrum of the oxidised species; however, this also overlaps with the ground state species and therefore an assignment is difficult to make. The TRIR spectrum of NDIS - PTZ is dominated by a relatively broad band at $1634 \mathrm{~cm}^{-1}$ and a ground state depletion at $1689 \mathrm{~cm}^{-1}$. In the TRIR, the fast component involves a depletion at $1623 \mathrm{~cm}^{-1}$ and a rise at $1670 \mathrm{~cm}^{-1}$. A further $200( \pm 100)$ ps component exists 
before the spectra return to baseline in ca. 3 ns. It can be concluded that the inclusion of sulfur substituents decreases the excited state lifetime when comparing NDIS $_{\mathbf{3}}$-PTZ to NDIS $\mathbf{S}^{-}$ PTZ.

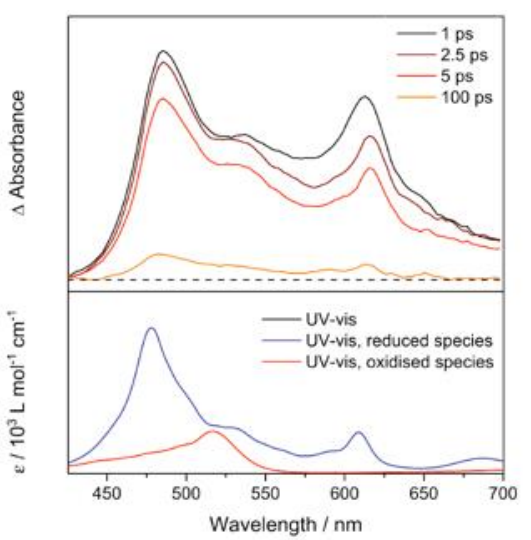

(a)

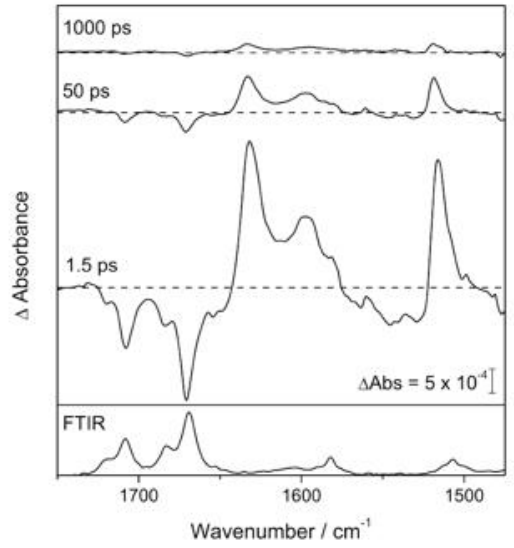

(d)

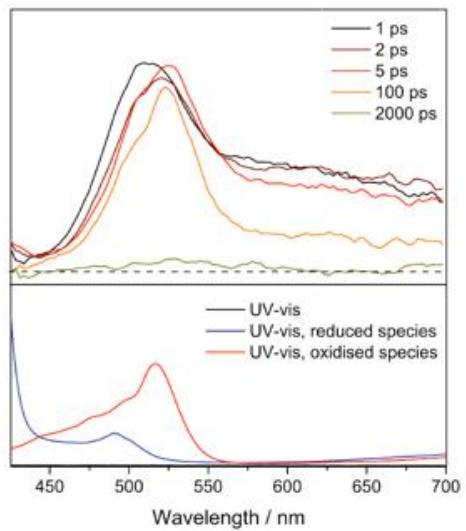

(b)

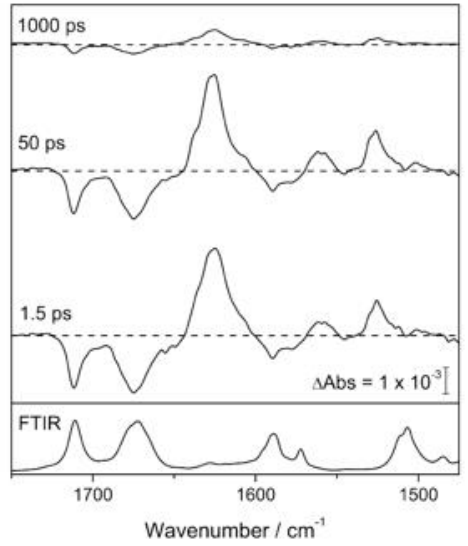

(e)

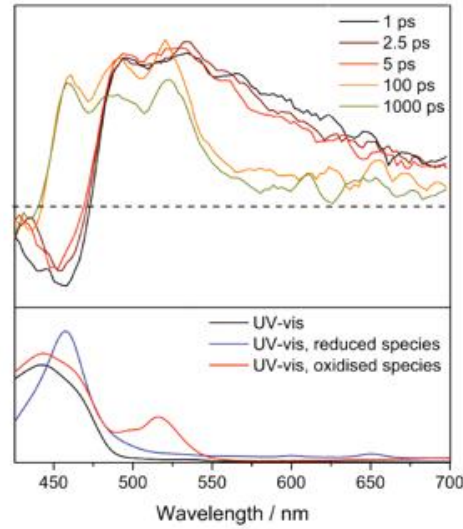

(c)

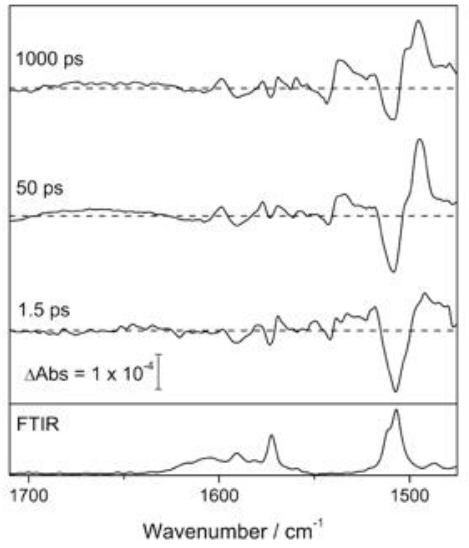

(f)

Figure 11. (a)-(c) Transient absorption and (d)-(f) TRIR spectra acquired in $\mathrm{CH}_{2} \mathrm{Cl}_{2}$ at selected time delays after photoexcitation with a $355 \mathrm{~nm}$ pulse. (a) NDIS $-\mathbf{P T Z}$, (b)reduced NIS $\mathbf{S}_{0}-\mathbf{P T Z}$,

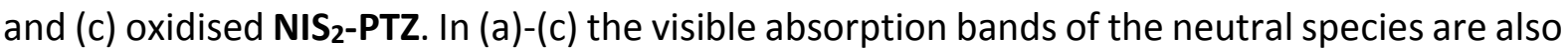
shown. In (d)-(f) the corresponding FT-IR spectra are also shown; (d) NDIS $\mathbf{S}_{0}-\mathbf{P T Z}$, (e) NIS $\mathbf{S}_{0}$-PTZ and (f) NIS - -PTZ.

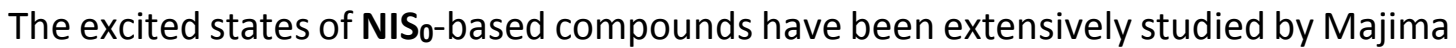
and co-workers, both in isolation and coupled to PTZ units. ${ }^{38-40,44-47}$ For example, NI-PTZ dyads have been examined ${ }^{38}$ where the linker that joins the donor and acceptor consisted of alkoxy chains or alkyl chains of various lengths. Long-lived charge transfer states were reported where bands characteristic of the radical anion of $\mathrm{NI}\left(\mathrm{NI}^{-}\right)$at $420 \mathrm{~nm}$ and the $\mathrm{PTZ}^{+}$band at 
$520 \mathrm{~nm}$ were used to assign the natures of these states. We have examined NIS0-PTZ, which is similar to the previously studied compounds, but differs by our use of a phenyl linker between the donor and acceptor. After the initial photoexcitation, the TA spectrum of NISPTZ shows a band at $c a .511 \mathrm{~nm}$ with a broad shoulder toward the red edge of the spectrum. A rapid (1 $( \pm 1)$ ps) decay is observed at $490 \mathrm{~nm}$, concurrent with an increase in intensity at $538 \mathrm{~nm}$; this manifests as a red-shift of the major band in the spectrum. A further process occurs with a lifetime of $23( \pm 8)$ ps, and this involves a depletion of the low-energy shoulder concurrent with a further growth of the major band at $523 \mathrm{~nm}$. Finally, the remaining bands decay to the ground state with a lifetime of $260( \pm 60)$ ps. As with the NDI compound above, the TA spectra of the final species appear to be a superposition of the electrochemically reduced and oxidised compound, indicating the formation of a charge-separated excited state. This reduced lifetime compared to the previously reported donor-acceptor assemblies likely stems from our use of a conjugated linker, compared to the more insulating alkyl or alkoxy linkers used previously. ${ }^{38} \mathrm{~A}$ new feature is the presence of a broad band stretching to the low-energy edge of the window, which is present in neither the oxidised nor the reduced species. The short-lived component at $490 \mathrm{~nm}$ may be due to the initially formed $\mathrm{NI}$ * excited state and a similar state for photoexcited 1,8-naphthalimide has previously been reported at $475 \mathrm{~nm} .{ }^{48}$ Thus, the $23 \mathrm{ps}$ spectral shift may represent the charge transfer process leading to the $\mathrm{NI}^{--}-\mathrm{PTZ}+{ }^{+}$state. The TRIR spectrum of $\mathbf{N I S}_{0}-\mathbf{P T Z}$ shows depletions of the parent bands and a number of new transient bands shifted to lower energy. As with NDISo-PTZ an initial (2 ( \pm 1$)$ ps) growth of the transient bands is observed. A further process can be fitted with a timeconstant of $c a .30$ ps, which spectrally involves a narrowing of the band at $1627 \mathrm{~cm}^{-1}$ and an increase in the intensity of bands at 1562 and $1529 \mathrm{~cm}^{-1}$. The latter band is close to an IR band of [methyl-PTZ] ${ }^{+}$, which has been reported at $1536 \mathrm{~cm}^{-1} .{ }^{49}$ The final bands are again consistent with the electrochemically reduced species (Figure S9). Lastly, the bands appear to return to the baseline over ca. $250( \pm 30)$ ps, consistent with observations from TA.

The TA spectrum of $\mathbf{N I S}_{\mathbf{2}}$-PTZ reveals similar behaviour to that of $\mathbf{N I S}_{\mathbf{0}}-\mathbf{P T Z}$ (Table 4) with the observed spectra matching well with the UV-visible absorptions of photochemically reduced and oxidised $\mathbf{N I S}_{2}-\mathbf{P T Z}$, again indicating that charge-transfer is taking place. The corresponding TRIR spectra show several low-intensity transient bands and a global fit of the data again indicates two processes on the picosecond timescale (see Table 4). Although the signal is low, the appearance of obvious new bands on a $30( \pm 10)$ ps timescale at 1598,1536 , 
1501 , and $1495 \mathrm{~cm}^{-1}$ suggests a significant change in the nature of the states probed. The nsTRIR experiment confirms the transient bands decay with a lifetime of $1.0( \pm 0.1) \mathrm{ns}$, however, a small amount (ca. 10\%) intensity is retained and a further decay with a lifetime of $5.0( \pm 1.0)$ ns is observed. On the faster timescale the formation we tentatively assign to the initially formed state to be localized (i.e. not charge-separated), with the 30 ps process representing charge-separation. This is also supported by the appearance of the band at $1535 \mathrm{~cm}^{-1}$, which shows a clear analogue in the photochemically oxidised spectrum (Figure S9).

The time-resolved spectroscopic studies presented above clearly point toward complex photophysical behaviours. However, several conclusions can be drawn. Primarily, it is evident from comparing the excited-state spectra of the PTZ-appended compounds with the photochemically generated species, that charge-separation takes place and is involved as the final state for all donor-acceptor dyads. Previous studies of NI-PTZ donor-acceptor dyads have revealed charge-separation to occur on the timescale of tens of picoseconds ${ }^{38}$ and this is consistent with our observations for the PTZ-appended molecules. For NIS $\mathbf{S}_{0}$-PTZ and NIS $\mathbf{S}_{2}$ PTZ, potential marker bands for charge transfer have also been identified in the TRIR and TA spectra. Furthermore, intersystem crossing (ISC) is expected to play a significant role, as it has previously been shown that the ${ }^{1} \mathrm{NI}^{*}$ state of NI-PTZ donor-acceptor dyads rapidly decays to

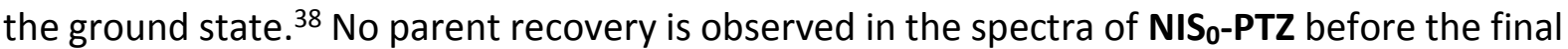
decay, which would suggest that ISC is rapid. This is in contrast to NDISo-PTZ, which shows significant parent recovery across all timescales. Finally, the presence of multiexponential excited-state decay pathways likely reflects that back-electron transfer is sensitive to factors such as exciplex formation. ${ }^{46}$

\section{Conclusions}

The most complete electrochemical characterisation to date of a series of thionated NDIs, NIs, and NDI/NI-phenothiazine dyads is reported. It has revealed thionation of NDIs as a straightforward method of manipulating/tuning the energy levels of the frontier orbitals. Each successive sulfur substitution caused a spectral redshift of $\sim 45 \mathrm{~nm}$, representing a decrease in the HOMO-LUMO gap. Cyclic voltammetry measurements indicated each thionation caused an anodic shift in the reduction potentials by approximately $150 \mathrm{mV}$, with DFT data confirming increased electron affinity is due to a decrease in LUMO energy. As chromophores, the thionated NDIs behave very similarly to their parent NDI; the 
bathochromic shifts caused by thionation are preserved upon reduction to stable radical species and when reduced again to form stable dianions.

DFT calculated molecular orbitals in tandem with spectroelectrochemistry confirmed that coupling of phenothiazine to NDIs and NI forms donor-acceptor complexes that are separated by a non-conjugated phenylene bridge. Thionation improves the electron acceptor ability of NDIs and NIs in a donor-acceptor complex without affecting the electron donor ability, allowing for precise control of the HOMO-LUMO separation. Complete thionation of the NI-PTZ dyads proved possible, and resulted in a pronounced effect of the electron accepting characteristics. An anodic shift in reduction potential alongside a $90 \mathrm{~nm}$ bathochromic shift in absorption maximum into the visible spectrum greatly improves the capacity of NIs for use in organic electronic applications with the optical gap of NDIS $\mathbf{S}_{\mathbf{3}}$-PTZ (0.96 eV) unprecedentedly low for this class of compound. Lastly, photoinduced chargeseparation was observed for these NDI and NI dyads, supported by data obtained from the transient spectra of the photoexcited compounds, indicating that thionation of the acceptor NDI or NI moiety leads to reduced lifetimes of the excited states.

\section{Acknowledgements}

We thank the Engineering and Physical Sciences Research Council (EP/K01773X/1) and the Leverhulme Trust (RPG-2014-317) for support; NRC gratefully acknowledges receipt of a Royal Society Wolfson Merit Award.

\section{Supporting Information Available.}

Detailed of synthetic procedures and characterisation data for all new compounds. Details of crystallographic structural refinement, additional UV-vis and EPR data for all the oxidation and reduction processes, further details of DFT calculations and additional figures for TA and TRIR experiments. CCDC-1500966 (NIS - PTZ), CCDC-1500960 (NIS - PTZ), CCDC-1500961

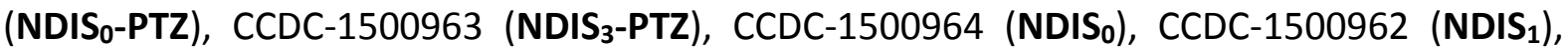
CCDC-1500965 (trans-NDIS 2 ) contain the supplementary crystallographic data for this paper.

\section{Notes and references}

1. J.R. Durrant, S.A. Haque and E. Palomares, Chem. Commun., 2006, 3279-3289.

2. D. Gust, T.A. Moore and A.L. Moore, Acc. Chem. Res. 2001, 34, 40-48. 
3. L. Flamigni, J-P. Collin and J-P. Sauvage, Acc. Chem. Res. 2008, 41, 857-871.

4. M. Hissler, J.E. McGarrah, W.B. Connick, D.K. Geiger, S.D. Cummings and R. Eisenberg, R. Coord. Chem. Rev., 2000, 208, 115-137.

5. S.V. Bhosale, C.H. Jani, and S.J. Langford, Chem. Soc. Rev. 2008, 37, 331-342.

6. M. Sommer, J. Mater. Chem. C 2014, 2, 3088-3098.

7. X. Zhan, A. Facchetti, S. Barlow, T.J. Marks, M.A. Ratner, M. R. Wasielewski and S.R. Marder, Adv. Mater. 2011, 23, 268-284.

8. Y. Zhao, Y. Guo and Y. Liu, Adv. Mater. 2013, 25, 5372-5391.

9. J.G. Laquindanum, H.E. Katz, A. Dodabalapur and A.J. Lovinger, J. Am. Chem. Soc. 1996, 118, 11331-11332.

10. Z. Chen, Y. Zheng, H. Yan and A. Facchetti, J. Am. Chem. Soc. 2009, 131, 8-9.

11. M. Borgström, N. Shaikh, O. Johansson, M.F. Anderlund, S. Styring, B. Åkermark, A. Magnuson and L. Hammarström, J. Am. Chem. Soc. 2005, 127, 17504-17515.

12. F. Chaignon, F. Buchet, E. Blart, M. Falkenstrom, L. Hammarstrom and F. Odobel, New J. Chem. 2009, 33, 408-416.

13. E. lengo, G.D. Pantos, J.K.M. Sanders, M. Orlandi, C. Chiorboli, S. Fracasso and F. Scandola, Chem. Sci. 2011, 2, 676-685.

14. V.L. Gunderson, A. L. Smeigh, C.H. Kim, D.T. Co and M.R. Wasielewski, J. Am. Chem. Soc. 2012, 134, 4363-4372.

15. N. Sakai, R. Bhosale, D. Emery, J. Mareda and S. Matile, J. Am. Chem. Soc. 2010, 132, 69236925.

16. S.T. Schneebeli, M. Frasconi, Z. Liu, Y. Wu, D.M. Gardner, N.L. Strutt, C. Cheng, R. Carmieli, M.R. Wasielewski and J.F. Stoddart, Angew. Chem. Int. Ed. 2013, 52, 13100-13104.

17. R.E. Dawson, A. Hennig, D.P. Weimann, D. Emery, V. Ravikumar, J. Montenegro, T. Takeuchi, S. Gabutti, M. Mayor, J. Mareda, C.A. Schalley and S. Matile, Nat. Chem. 2010, 2, 533-538.

18. S. Guha and S. Saha, J. Am. Chem. Soc. 2010, 132, 17674-17677.

19. M. Pan, X-M. Lin, G-B. Li and C-Y. Su, Coord. Chem. Rev. 2011, 255, 1921-1936.

20. T.B. Schon, B.T. McAllister, P-F. Li and D.S. Seferos, Chem. Soc. Rev., 2016, 45,6345-6404.

21. S. Erten and S. Icli, Inorg. Chim. Acta, 2008, 361, 595.

22. Y. Lin, Y. Li and X. Zhan, Chem. Soc. Rev., 2012, 41, 4245-4272.

23. N. Sakai, J. Mareda, E. Vauthey and S. Matile, Chem. Commun. 2010, 46, 4225-4237.

24. S-L. Suraru and F. Würthner, Angew. Chem. Int. Ed. 2014, 53, 7428-7448.

25. J. Quinn, Y. Zheng, Z. Chen, H. Usta, C. Newman, H. Yan and A. Facchetti, 2011 US Patent Appl. US 8440828 B2. 
26. L.M. Kozycz, C. Guo, J.G. Manion, A.J. Tilley, A.J. Lough, Y. Li and D.S. Seferos, J. Mater. Chem. C, 2015, 3, 11505-11515.

27. F.S. Etheridge, R. Fernando, J.A. Golen, A.L. Rheingold and G. Sauve, RSC Adv. 2015, 5, 4653446539 .

28. A..J. Tilley, R. D. Pensack, T. S. Lee, B. Djukic, G.D. Scholes and D.S. Seferos, J. Phys. Chem. C 2014, 118, 9996-10004.

29. A.J. Tilley, C. Guo, M.B. Miltenburg, T.B. Schon, H. Yan, Y. Li and D.S. Seferos, Adv. Funct. Mater., 2015, 22, 3321-3329.

30. B.A. Llewellyn, E.S. Davies, C.R. Pfeiffer, M. Cooper, W. Lewis and N.R. Champness, Chem. Commun., 2016, 52, 2099-2102.

30. E.A. Weiss, M.J. Ahrens, L. E. Sinks, A. V. Gusev, M. A. Ratner and M. R. Wasielewski, J. Am. Chem. Soc. 2004, 126, 5577-5584.

32. G. Andric, J.F. Boas, A.M. Bond, G.D. Fallon, K.P. Ghiggino, C.F. Hogan, J. A. Hutchison, M. A-P. Lee, S.J. Langford, J.R. Pilbrow, G.J. Troup and C. P. Woodward, Aust. J. Chem., 2004, 57, 10111019.

33. B.M. Aveline, S. Matsugo and R.W. Redmond, J. Am. Chem. Soc., 1997, 119, 11785-11795.

34. S. Green and M.A. Fox, J. Phys. Chem., 1995, 99, 14752-14757.

35. J.E. Rogers and L.A. Kelly, J. Am. Chem. Soc., 1999, 121, 3854-3861.

36. I.V. Sazanovich, M.A.H. Alamiry, J. Best, R.D. Bennett, O. V. Bouganov, E. S. Davies, V. P. Grivin, A. J. H. M. Meijer, V. F. Plyusnin, K. L. Ronayne, A. H. Shelton, S. A. Tikhomirov, M. Towrie and J A. Weinstein, Inorg. Chem., 2008, 47, 10432-10445.

37. P. Ganesan, J. Baggerman, H. Zhang, E.J.R. Sudhölter and H. Zuilhof, J. Phys. Chem. A, 2007, 111, 6151-6156.

38. D.W. Cho, M. Fujitsuka, A. Sugimoto, U.C. Yoon, P. S. Mariano and T. Majima, J. Phys. Chem. $B, 2006,110,11062-11068$.

39. G. Jones II and S. Kumar, J. Photochem. Photobio. A, 2003, 160, 139-149.

40. T. Takada, K. Kawai, M. Fujitsuka and T. Majima, Proc. Natl. Acad. Sci. USA 2004, 101, $14002-$ 14006.

41. Y. Mori, Y. Sakaguch and H. Hayashi, J. Phys. Chem. A, 2002, 106, 4453-4467.

42. N. Banerji, S.V. Bhosale, I. Petkova, S.J. Langford and E. Vauthey, Phys. Chem. Chem. Phys., 2011, 13, 1019-1029.

43. D. Villamaina, S.V. Bhosale, S. J. Langford and E. Vauthey, Phys. Chem. Chem. Phys., 2013, 15, 1177-1187. 
44. D.W. Cho, M. Fujitsuka, U.C. Yoon and T. Majima, J. Photochem. Photobio. A, 2007, 190, 101109.

45. D.W. Cho, M. Fujitsuka, A. Sugimoto and T. Majima, J. Phys. Chem. A, 2008, 112, 7208-7213.

46. D.W. Cho, M. Fujitsuka, K.H. Choi, M. J. Park, U. C. Yoon and T. Majima, J. Phys. Chem. B, 2006, $110,4576-4582$.

47. D.W. Cho, M. Fujitsuka, U. Chan Yoon and T. Majima, Phys. Chem. Chem. Phys., 2008, 10, 43934399.

48. A. Samanta and G. Saroja, J. Photochem. Photobio. A, 1994, 84, 19-26. 